\title{
Asteroid observation and landing trajectories using
}

\section{invariant manifolds}

\author{
E. Herrera-Sucarrat ${ }^{1}$ and P.L. Palmer ${ }^{2}$ \\ Surrey Space Centre, University of Surrey, Guildford, GU2 7XH, UK \\ R.M. Roberts ${ }^{3}$ \\ Mathematics Department, University of Surrey, Guildford, GU2 7XH, UK.
}

In this paper a study of the equilibrium points of a rotating non-spherical asteroid is performed with special emphasis on the equilibria aligned with the longest axis of the body. These equilibrium points have the same spectral behaviour as the collinear Lagrange points of the Restricted Three Body Problem (RTBP), saddle-centres, and therefore unstable and stable invariant manifolds can be computed. The invariant manifolds of the equilibrium point or periodic orbits around it, which are fuel-free trajectories, can approach the surface of the asteroid, orbit around it for different amounts of time, and even impact on it. This paper studies the dependence of the existence of fuel-free trajectories to the surface of the asteroid from the equilibrium point on the shape and rotation rate of the body. A possible manoeuvre to orbit the asteroid to observe it and later achieve vertical landing is proposed. The theory developed is then applied to asteroid 4660 Nereus, for which an approach, observation phase and landing manoeuvre is designed.

\footnotetext{
${ }^{1}$ PhD student at Surrey Space Centre and Mathematics Department, University of Surrey, Guildford, GU2 7XH, UK. e.herrerasucarrat@surrey.ac.uk

2 Reader at Surrey Space Centre, University of Surrey, Guildford, GU2 7XH, UK. p.palmer@surrey.ac.uk

${ }^{3}$ Head of Mathematics Department, University of Surrey, Guildford, GU2 7XH, UK. m.roberts@surrey.ac.uk
} 


\section{Nomenclature}

$I_{x x} \quad=$ principal moments of inertia in $x$ direction

$I_{y y} \quad=$ principal moments of inertia in $y$ direction

$I_{z z} \quad=$ principal moments of inertia in $z$ direction

$\alpha_{x}, \alpha_{y}=$ shape parameters (moments of inertia ratios)

$\omega=$ angular velocity of asteroid in dimensionless units

$M \quad=$ asteroid's mass

$R=\sqrt{I_{z z} / M}$

$a, b, c=$ semi axes ellipsoid

$\rho \quad=$ density

$\Phi_{i} \quad=$ internal potential

$\Phi_{e} \quad=$ external potential

$\bar{R} \quad=$ radius of the circumscribing sphere

$\mathcal{C}=$ Jacobi constant

$\phi \quad=$ position angle on the ellipsoid measured from the semi-major axis

$\alpha=$ angle of the velocity vector and the tangent of the ellipsoid at angle $\phi$

\section{Introduction}

The last two decades have witnessed several missions to asteroids and comets including NASA's NEAR [1], Deep Impact [2], Stardust [3] and Dawn [4], ESA's Rosetta [5] and JAXA's Hayabusa [6]. There are several reasons for this current interest in the smallest bodies of the Solar System. First, the scientific community believes that they represent the remnant debris of the first years of the formation of the Solar System and also may yield information of how our planet was created. Secondly, some asteroids, specially the ones near the Earth, can be explored with lower mission velocities than any other body, which makes them very attractive as targets for scientific missions or for sources of extraterrestrial material. Finally, asteroids and comets might pose a serious threat to our planet when they approach the Earth, becoming NEOs (Near Earth Objects).

Designing an asteroid landing trajectory is a complicated and challenging problem. The main 
issue is the lack of knowledge about its shape, gravitational potential and rotational dynamics. Asteroids and comets are characterised by very irregular shapes, which makes mathematical models that reduce the bodies to point masses unsuitable for planning missions.

In this paper, the model used for the gravitational potential of the non-spherical body is an expansion up to second order in terms of spherical Bessel functions and spherical harmonics that matches smoothly with the well known MacCullagh's formula at a given spherical boundary, hence satisfying both Poisson's and Laplace's equation. This expansion of the potential can be used to model asteroids for which only the mass and moments of inertia are known, and at the same time allows for a general study of the dynamics in terms of the potential coefficients given by the mass and inertia moments of the asteroid.

The fact that asteroids are not point masses but elongated bodies leads to a richer dynamics around them compared to the simple Kepler's problem. The dynamics of massless particles around an elongated asteroid are modelled using the Restricted Full Two Body Problem (RF2BP). This mathematical model has similarities with the well known Restricted Three Body Problem (RTBP). Relative equilibrium points exist in their vicinity [8-10], together with stable and unstable periodic orbits around them. As in the RTBP, two of the equilibrium points around an asteroid have a unstable saddle-centre behaviour, and therefore unstable periodic orbits around them with stable and unstable invariant manifolds exist $[11,14]$. Moreover, the theory developed in the RTBP about the dynamics around the collinear Lagrange points can be adapted to this problem [18, 19]. In the RTBP the invariant manifolds of periodic orbits around Lagrange points approach the secondary body but do not come near the primary body. In the RF2BP, however, the invariant manifolds of the unstable periodic orbits might approach or intersect the body, depending upon the shape and the asteroid's rotation rate. These manifolds then offer fuel-free trajectories that approach or depart from the asteroid at low relative velocities that can be used for landing and taking off for sample-return missions. Invariant manifolds of periodic orbits have been used in [11] to transfer from higher orbits to lower orbits of Vesta.

In recent decades, lots of research has been done applying the dynamical model of the RTBP to real missions, exploiting the natural dynamics of invariant manifolds to explore the Universe 
without high consumptions of fuel. In this paper, the same idea is applied to asteroid missions. The possibility of approaching and landing on an asteroid using the natural dynamics around unstable equilibrium points given by the non-spherical nature of the asteroid is studied.

In this paper, the dependence of the dynamical environment of non-spherical bodies on the shape (moments of inertia) and rotation period will be studied. The paper focuses on the computation and study of the behaviour of the invariant manifolds of equilibrium points and of periodic orbits depending on the parameters of the problem and the application of the manifolds to develop a method for landing on asteroids using the natural dynamics around them. The structure of the paper is as follows. In section II a summary of how to compute the gravitational potential used is given. In section III the dynamical environment of an asteroid is studied determining the behaviour of the manifolds depending on the rotation and shape of the body. Finally, in section IV a possible landing manoeuvre is described, with the example of asteroid Nereus.

\section{Expansion of the gravitational potential up to second order}

For the majority of asteroids discovered there is not enough information about their shape to develop a good approximation of their gravitational potential. For those asteroids of which only the triaxial axes and a mean density are known, an approximation of the mass and moments of inertia can be computed (assuming a triaxial shape and constant density). With this information an expansion up to second order in terms of spherical Bessel functions and spherical harmonics can be derived, allowing for a very simple expression of the gravitational potential which is easy to compute and is dynamically equivalent to the gravitational potential of a constant density ellipsoid, but that does not require the constant evaluation of elliptic integrals.

Let $\Phi(x, y, z)$ the potential that the point $P=(x, y, z)$ experiences due to the attraction of a non-spherical body. The potential $\Phi(x, y, z)$ and the three components of the force $\left(\frac{\partial \Phi}{\partial x}, \frac{\partial \Phi}{\partial y}, \frac{\partial \Phi}{\partial z}\right)$ exist at all points and are continuous throughout space. Moreover, the gravitational potential $\Phi$ must satisfy Laplace's equation for regions away from the attracting matter, $\nabla^{2} \Phi=0$, and Poisson's equation for regions within the attracting matter, $\nabla^{2} \Phi=-4 \pi \rho G$, [22].

A possible way to solve for the potential of a particular mass distribution is to solve inde- 
pendently both equations and impose that the solutions and their derivatives match at a given boundary.

\section{A. The external potential of a rigid body: solution of Laplace's equation}

It is well known that outside a spherical boundary that contains the mass of the body, the solution of Laplace's equation, which we call the external potential, can be expressed using spherical harmonics [21, 23]:

$$
\Phi_{e}(r, \theta, \varphi)=\frac{G}{R} \sum_{n=0}^{\infty}\left(\frac{R}{r}\right)^{n+1} \sum_{m=0}^{n}\left(a_{n m} P_{n}^{m}(\cos (\theta)) \cos (m \varphi)+b_{n m} P_{n}^{m}(\cos (\theta) \sin (m \varphi))\right.
$$

where $(r, \theta, \varphi)$ are the spherical coordinates, $R$ the radius of the spherical boundary, $P_{n}^{m}(\cos \theta)$ the associated Legendre Polynomials and $a_{n m}$ and $b_{n m}$ the coefficients of the expansion,

$$
\begin{aligned}
& a_{n 0}=\iiint_{V}\left(\frac{r}{R}\right)^{n} P_{n}(\cos \theta) \rho(r, \theta, \varphi) \mathrm{d} V, \\
& a_{n m}=2 \frac{(n-m) !}{(n+m) !} \iiint_{V}\left(\frac{r}{R}\right)^{n} C_{n m}(\theta, \varphi) \rho(r, \theta, \varphi) \mathrm{d} V, \text { for } m \neq 0 \\
& b_{n m}=2 \frac{(n-m) !}{(n+m) !} \iiint_{V}\left(\frac{r}{R}\right)^{n} S_{n m}(\theta, \varphi) \rho(r, \theta, \varphi) \mathrm{d} V, \text { for } m \neq 0 .
\end{aligned}
$$

computed knowing the body's density $\rho$.

\section{MacCullagh's Formula in cartesian coordinates}

If the origin of the coordinate system coincides with the centre of gravity of the non-spherical body, then $a_{10}=a_{11}=b_{11}=0$. If the frame coordinates are aligned with the principal inertia axes of the body, then the products of inertia are zero and $a_{20}=\frac{1}{2 R^{2}}\left(I_{x x}+I_{y y}-2 I_{z z}\right), a_{21}=0$, $a_{22}=\frac{1}{4 R^{2}}\left(I_{y y}-I_{x x}\right), b_{21}=0$ and $b_{22}=0$, where $I_{x x}, I_{y y}$ and $I_{z z}$ are the principal moments of inertia in the $x, y$ and $z$ direction.

Using these coefficients, the potential up to second order can be written as

$\Phi_{e}(x, y, z)=\frac{G M}{\sqrt{x^{2}+y^{2}+z^{2}}}\left[1+\frac{1}{2\left(x^{2}+y^{2}+z^{2}\right)}\left(\operatorname{tr}\left(\bar{I}_{3}\right)-\frac{3}{x^{2}+y^{2}+z^{2}}\left(\bar{I}_{x x} x^{2}+\bar{I}_{y y} y^{2}+\bar{I}_{z z} z^{2}\right)\right)\right]$,

where $I_{3}$ represents the diagonal matrix with elements $\left(I_{x x}, I_{y y}, I_{z z}\right)$ and the bar represents the variable divided by the total mass $M$ [24]. This formula is known as MacCullagh's formula and it 
gives the external potential to second order of a body of mass $M$ and moments of inertia $I_{x x}, I_{y y}$ and $I_{z z}$.

\section{B. The internal potential of a rigid body: solution of Poisson's equation}

Following the theory developed in [25, 26], the solution of Poisson's equation can be obtained by expanding the potential and density of the body in terms of a series of basis functions: spherical harmonics and spherical Bessel functions $\left(j_{n}\right)$. The internal potential can be expanded as

$$
\Phi_{i}(r, \theta, \varphi)=\frac{G}{R} \sum_{l=0}^{\infty} \sum_{n=0}^{\infty} \sum_{m=0}^{n} j_{n}\left(\frac{\alpha_{l n} r}{R}\right) P_{n}^{m}(\cos \theta)\left(A_{l n m} \cos (m \varphi)+B_{l n m} \sin (m \varphi)\right),
$$

where $R$ is the radius of the circumscribing circle, the coefficients $A_{l n m}$ and $B_{l n m}$ depend on the body, and $\alpha_{l n}$ are dimensionless coefficients which are determined by imposing that the internal and external potential are identical when $r=R$ and $\lim _{r \rightarrow R^{-}} \frac{\partial \Phi_{i}}{\partial r}=\lim _{r \rightarrow R^{+}} \frac{\partial \Phi_{e}}{\partial r}$. This will guarantee that the potential function defined as the external potential $\Phi_{e}$ when $r \geq R$ and the internal potential $\Phi_{i}$ when $r \leq R$ and the force, $\left(\frac{\partial \Phi}{\partial x}, \frac{\partial \Phi}{\partial y}, \frac{\partial \Phi}{\partial z}\right)$, exits everywhere and are continuous.

In order to determine the coefficients $\alpha_{n l}$ it is easier if the external potential is also written in terms of spherical Bessel functions. It then has the following expression

$$
\Phi_{e}(r, \theta, \varphi)=\frac{G}{R} \sum_{l=0}^{\infty} \sum_{n=0}^{\infty} \sum_{m=0}^{n} j_{n}\left(\alpha_{l n}\right)\left(\frac{R}{r}\right)^{n+1} P_{n}^{m}(\cos \theta)\left(A_{l n m} \cos (m \varphi)+B_{l n m} \sin (m \varphi)\right) .
$$

Therefore, by imposing that $\lim _{r \rightarrow R^{-}} \frac{\partial \Phi_{i}}{\partial r}=\lim _{r \rightarrow R^{+}} \frac{\partial \Phi_{e}}{\partial r}$ and by using the properties of the spherical Bessel functions, it is possible to see that $\alpha_{n l}$ are the solutions of $j_{n-1}\left(\alpha_{l n}\right)=0$.

Using equation (6) in Poisson's equation, it is possible to find an analytic expression for the density distribution of the body in terms of the spherical harmonics and spherical Bessel functions

$$
\rho(r, \theta, \varphi)=\sum_{l=0}^{\infty} \sum_{n=0}^{\infty} \sum_{m=0}^{n} \alpha_{l n}^{2} j_{n}\left(\frac{\alpha_{l n} r}{R}\right) P_{n}^{m}(\cos \theta)\left(A_{l n m} \cos (m \varphi)+B_{l n m} \sin (m \varphi)\right) .
$$

Due to the properties of orthogonality of the basis functions, the coefficients $A_{\operatorname{lnm}}$ and $B_{\operatorname{lnm}}$ can be computed,

$$
\begin{aligned}
A_{l n 0} & =\frac{2(2 n+1)}{\alpha_{l n}^{2}\left(j_{n}\left(\alpha_{l n}\right)\right)^{2}} \iiint_{V} j_{n}\left(\frac{\alpha_{l n} r}{R}\right) P_{n}^{0}(\cos \theta) \rho(r, \theta, \varphi) \mathrm{d} V, \\
A_{l n m} & =\frac{4(2 n+1)}{\alpha_{l n}^{2}\left(j_{n}\left(\alpha_{l n}\right)\right)^{2}} \frac{(n-m) !}{(n+m) !} \iiint_{V} j_{n}\left(\frac{\alpha_{l n} r}{R}\right) P_{n}^{m}(\cos \theta) \cos (m \varphi) \rho(r, \theta, \varphi) \mathrm{d} V, \\
B_{l n m} & =\frac{4(2 n+1)}{\alpha_{l n}^{2}\left(j_{n}\left(\alpha_{l n}\right)\right)^{2}} \frac{(n-m) !}{(n+m) !} \iiint_{V} j_{n}\left(\frac{\alpha_{l n} r}{R}\right) P_{n}^{m}(\cos \theta) \sin (m \varphi) \rho(r, \theta, \varphi) \mathrm{d} V .
\end{aligned}
$$


Due to the fact that both expressions for the external potential, equations (1) and (7), are the same, the relationships between their coefficients are

$$
\begin{aligned}
& a_{n m}=\sum_{l=0}^{\infty} j_{n}\left(\alpha_{l n}\right) A_{l n m} \\
& b_{n m}=\sum_{l=0}^{\infty} j_{n}\left(\alpha_{l n}\right) B_{l n m} .
\end{aligned}
$$

\section{The external-internal potential matching: the choice of the coefficients}

For asteroids such that only the mass $M$ and the principal moments of inertia $I_{x x}, I_{y y}$ and $I_{z z}$ are known, the external potential up to second order can be easily computed using MacCullagh's formula. In order to compute the internal potential the coefficients $A_{l n m}$ and $B_{l n m}$ have to be calculated using only the information known.

From the computation of the coefficients of MacCullagh's formula, it is known that $\frac{a_{00}}{M}=1$, $\frac{a_{20}}{M}=\frac{1}{2 M R^{2}}\left(I_{x x}+I_{y y}-2 I_{z z}\right)=\frac{1}{2 R^{2}}\left(I_{x x}^{-}+I_{y y}^{-}-2 I_{z z}^{-}\right)$and $\frac{a_{22}}{M}=\frac{1}{4 M R^{2}}\left(I_{y y}-I_{x x}\right)=\frac{1}{4 R^{2}}\left(I_{y y}^{-}-I_{x}^{-} x\right)$. Therefore using that $\Phi_{i}(R)=\Phi_{e}(R)$, an expansion up to second order of the internal potential will have coefficients satisfying

$$
\begin{aligned}
1 & =\sum_{l=0}^{\infty} A_{l 00} j_{0}\left(\alpha_{0 l}\right), \\
\frac{1}{2 R^{2}}\left(I_{x x}^{-}+\bar{I}_{y y}^{-}-2 \overline{I_{z z}}\right) & =\sum_{l=0}^{\infty} A_{l 20} j_{2}\left(\alpha_{2 l}\right), \\
\frac{1}{4 R^{2}}\left(\bar{I}_{y y}^{-}-I_{x x}^{-}\right) & =\sum_{l=0}^{\infty} A_{l 22} j_{2}\left(\alpha_{2 l}\right) .
\end{aligned}
$$

plus the equation that guarantees that the density of the body at the spherical boundary must vanish

$$
\lim _{r \rightarrow R} \rho(r, \theta, \varphi)=0 \quad \forall \theta \forall \varphi
$$

This condition on the density guarantees that the second derivative of the potential is continuous, and hence the dynamics of equilibrium points are be continuous across the circumscribing sphere. As an expansion up to second order in terms of the inertia moments is used, the coefficients $B_{l n m}$ will satisfy $\sum_{l=0}^{\infty} B_{l n m} j_{n}\left(\alpha_{n l}\right)=b_{n m}=0$ and therefore are always zero for all $l, n=0,1,2$ and $m=0,1,2$. 
In order to determine the coefficients one should compute the integrals of equations (9), (10) and (11), which requires knowledge of the density of the body $\rho$. However, as only the mass and moments of inertia of this body are known, theses integrals cannot be evaluated. Therefore, there are infinite parameters to determine but only four equations. Hence the expansion of the potential will have free parameters. In order to solve the problem, the coefficients $A_{l n m}$ and $B_{l n m}$ are chosen in such a way that they satisfy equations $(14),(15),(16)$ and (17). Other choices of the parameters that make the potential satisfy the required equations will be valid as well and will give rise to different density distributions of the body. In this paper

$$
\begin{aligned}
& A_{l 00}=\frac{\alpha_{00}^{2}}{j_{0}\left(\alpha_{l 0}\right)\left(\alpha_{00}^{2}-\alpha_{l 0}^{2}\right)} \frac{g(l)}{\sum_{n=1}^{\infty} g(n)} \quad l \geq 1 \\
& A_{l 20}=\frac{\frac{1}{2 R^{2}}\left(I_{x x}^{-}+I_{y y}^{-}-2 I_{z z}^{-}\right) \alpha_{12}^{2}}{j_{2}\left(\alpha_{l 2}\right)\left(\alpha_{12}^{2}-\alpha_{l 2}^{2}\right)} \frac{g(l)}{\sum_{n=1}^{\infty} g(n)} \quad l \geq 2 \\
& A_{l 22}=\frac{\frac{1}{4 R^{2}}\left(I_{y y}^{-}-I_{x x}^{-}\right) \alpha_{12}^{2}}{j_{2}\left(\alpha_{l 2}\right)\left(\alpha_{12}^{2}-\alpha_{l 2}^{2}\right)} \frac{g(l)}{\sum_{n=1}^{\infty} g(n)} \quad l \geq 2
\end{aligned}
$$

and

$$
\begin{aligned}
& A_{000}=-\frac{\sum_{l=1}^{\infty} \alpha_{l 0}^{2} j_{2}\left(\alpha_{l 0}\right) A_{l 00}}{\alpha_{00}^{2} j_{0}\left(\alpha_{00}\right)}, \\
& A_{120}=-\frac{\sum_{l=2}^{\infty} \alpha_{l 2}^{2} j_{2}\left(\alpha_{l 2}\right) A_{l 20}}{\alpha_{12}^{2} j_{2}\left(\alpha_{12}\right)}, \\
& A_{122}=-\frac{\sum_{l=2}^{\infty} \alpha_{l 2}^{2} j_{2}\left(\alpha_{l 2}\right) A_{l 22}}{\alpha_{12}^{2} j_{2}\left(\alpha_{12}\right)} .
\end{aligned}
$$

where $\sum_{n=1}^{\infty} g(n)$ has to be a convergent series. When implementing this potential on a computer, the expansion will be truncated at some order, and hence the series should converge fast enough to maintain the accuracy of the matching between the internal and external potential. The convergent series used is $1 / n^{10}$.

In this section, the coefficients of the internal potential expansion have been chosen in such a way that the internal and external potential match at the circumscribing sphere and such that the body has the appropriate mass and moments of inertia. Other choices of the coefficients that make the internal potential satisfy the same restrictions will be valid as well. Although different choices will give rise to different density distributions of the body, and therefore, different dynamics, the overall behaviour will be similar. 


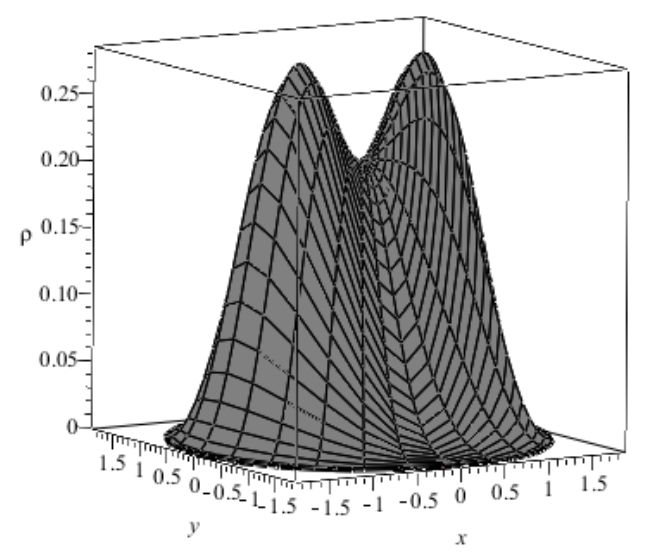

Fig. 1 Normalised density for asteroid Nereus given triaxial dimensions of $2 a=510 \mathrm{~m}, 2 b=330 \mathrm{~m}$ and $2 c=241 \mathrm{~m}$ and mean density of $2 \mathrm{gcm}^{-3}$.

\section{The dynamical environment of an elongated asteroid}

The Restricted Full Two Body Problem [8, 9, 13, 27, 28] describes the movement of a massless particle under the influence of a non-spherical body which is rotating uniformly around a principal axis. Using the expansion of the potential summarised in the previous section, it is possible to describe the dynamical environment of the body everywhere, up to second order.

Given a non-spherical body of mass $M$ uniformly rotating around the axis with maximum moment of inertia, a rotating frame of reference is used, centred at the body's centre of mass and always aligned with the principal axes of the body. The $x$-axis is aligned with the principal axis with minimum moment of inertia, the $z$-axis with the maximum moment of inertia and the $y$-axis completing the orthogonal frame. Then $I_{x x} \leq I_{y y} \leq I_{z z}$, and the angular velocity of the body can be written $\boldsymbol{\omega}=(0,0, \omega)$.

The equations of motion of a massless particle under the influence of the rotating body are

$$
\left.\begin{array}{rl}
\ddot{x}-2 \dot{y} \omega-x \omega^{2} & =\frac{\partial V}{\partial x} \\
\ddot{y}+2 \dot{x} \omega-y \omega^{2} & =\frac{\partial V}{\partial y} \\
\ddot{z} & =\frac{\partial V}{\partial z}
\end{array}\right\}
$$


where

$$
V(x, y, z)=\left\{\begin{array}{l}
\Phi_{i}, \text { if } r=\sqrt{x^{2}+y^{2}+z^{2}} \leq R, \\
\Phi_{e}, \text { if } r=\sqrt{x^{2}+y^{2}+z^{2}} \geq R
\end{array}\right.
$$

in cartesian coordinates.

In order to have dimensionless coordinates a fundamental unit of length and a fundamental unit of time are defined. If $I_{z z}=M \bar{R}^{2}, I_{x x}=\alpha_{x} M \bar{R}^{2}$ and $I_{y y}=\alpha_{y} M \bar{R}^{2}$ with $1 \geq I_{z z} \geq I_{y y} \geq I_{x x}$, the fundamental unit of length is $\tilde{r}=r / \bar{R}$ and the fundamental unit of time $\tilde{t}=n t$ where $n=\sqrt{G M / \bar{R}^{3}}$ the mean motion at distance $\bar{R}$.

Without the tildes, the non-dimensional equations of motion are the same as equation (24) with the following expressions for the potential

$$
\begin{aligned}
\Phi_{e}(x, y, z)= & \frac{1}{r}\left(1+\frac{1}{2 r^{2}}\left(1+\alpha_{x}+\alpha_{y}-\frac{3}{r^{2}}\left(\alpha_{x} x^{2}+\alpha_{y} y^{2}+z^{2}\right)\right)\right), \\
\Phi_{i}(x, y, z)= & \frac{\bar{R}}{R}\left(\sum_{l=0}^{\infty} \overline{A_{l 00} j_{0}}\left(\frac{\alpha_{l 0} r}{R / \bar{R}}\right)+P_{2}^{0}\left(\frac{z}{\sqrt{x^{2}+y^{2}}}\right) \sum_{l=0}^{\infty} A_{l 20}^{-} j_{2}\left(\frac{\alpha_{l 2} r}{R / \bar{R}}\right)+\right. \\
& \left.+P_{2}^{2}\left(\frac{z}{\sqrt{x^{2}+y^{2}}}\right)\left(\frac{x^{2}-y^{2}}{x^{2}+y^{2}}\right) \sum_{l=0}^{\infty} \overline{A_{l 22}^{-} j_{2}}\left(\frac{\alpha_{l 2} r}{R / \bar{R}}\right)\right),
\end{aligned}
$$

where $r=\sqrt{x^{2}+y^{2}+z^{2}}$, and the bar on the expansion coefficients means that they are divided by the mass $M$. This potential has been computed by normalising the variables and dividing it by $M n^{2} \bar{R}^{2}$. These equations have an integral of motion, the Jacobi constant with expression

$$
\mathcal{C}=\frac{1}{2}\left(\dot{x}^{2}+\dot{y}^{2}+\dot{z}^{2}\right)-\frac{1}{2} \omega^{2}\left(x^{2}+y^{2}\right)-V(x, y, z) .
$$

From now on, only the planar problem will be considered, i.e. the motion of the massless particle or spacecraft will be confined in the $x, y$ plane. This can be done due to the symmetry of the body given by the $x, y$ plane.

Using the equations given by the RF2BP model, the aim of this paper is to understand the role of the invariant manifolds in the dynamical environment of a non-spherical body, and how these manifolds can be used for mission planning and landing on asteroids.

The bodies considered in this paper have non-dimensional shape parameters $\alpha_{x}$ and $\alpha_{y}$, and rotation periods $T$ in hours. Therefore, to compute the non-dimensional angular velocity $\omega$ the mass and the fundamental unit of length are needed. For the computation of the mass, the body 
is assumed to be an ellipsoid with the required moments of inertia and constant density $2.5 \mathrm{~g} / \mathrm{cm}^{3}$.

Other densities can be considered, which will change the results slightly but the overall behaviour will be the same. The non-dimensional semi-major axes of the constant density ellipsoid are

$$
\begin{aligned}
a / \bar{R} & =\sqrt{\frac{5}{2}\left(1+\alpha_{y}-\alpha_{x}\right)}, \\
b / \bar{R} & =\sqrt{\frac{5}{2}\left(1+\alpha_{x}-\alpha_{y}\right)}, \\
c / \bar{R} & =\sqrt{\frac{5}{2}\left(-1+\alpha_{y}+\alpha_{x}\right)} .
\end{aligned}
$$

\section{A. Equilibrium points}

The equations of motion of the RF2BP with the internal and external potential can have relative equilibrium points aligned with the $x$ and $y$ axes (see figure 2). However, the only real equilibrium points are the ones outside the body. Due to the fact that the real density of the asteroid and its shape are not known, the gravitational potential used does not give information on where the surface of the body is. For simplicity, and in order to plot an approximate surface of the body, it will be assumed that the asteroid has the shape of an ellipsoid with semi-major axes given by equations (27), (28) and (29).

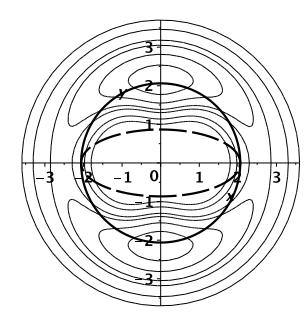

(a) $T=6 h$

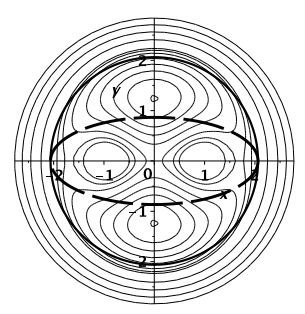

(b) $T=3 h$

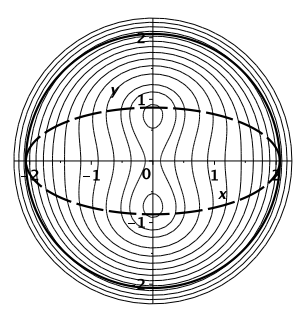

(c) $T=2 h$

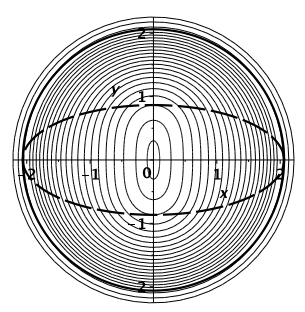

(d) $T=1.5 \mathrm{~h}$

Fig. 2 Zero velocity curves for a body with different rotation periods and shape parameters $\alpha_{x}=0.3$ and $\alpha_{y}=1$. The dashed line is the equatorial section of the ellipsoid that has the same moments of inertia and the circle is the spherical boundary where the potentials are matched. In 2(a) four equilibrium points exist outside of the body, in the region where the external potential is used. In 2 (b) the equilibrium points aligned with the long axis exist but are inside the body, and the ones aligned with the short axis are in the area of the internal potential. In 2(c) long axis equilibrium points do not exist and the short axis equilibria are inside the body. Finally, in 2(d) only an equilibrium point at the centre of the body exists. 


\section{Long axis equilibrium points}

Depending on the shape and rotation of the body there can exist two equilibrium points aligned with the $x$-axis (one at each side of the body) called the long axis equilibrium points, [10]. The equation of the long axis equilibrium point is

$$
\omega^{2}=\frac{1}{x_{e q}^{3}}\left(1+\frac{3}{2 x_{e q}^{2}}\left(1+\alpha_{y}-2 \alpha_{x}\right)\right)
$$

The minimum $x_{e q}$ allowed to have an external equilibrium point is at the surface of the body

$x_{e q}=\sqrt{\frac{5}{2}\left(1+\alpha_{y}-\alpha_{x}\right)}$. Then, $\omega_{*}^{2}=-\frac{4}{25} \frac{-8-8 \alpha_{y}+11 \alpha_{x}}{\left(-1-\alpha_{y}+\alpha_{y}\right)^{2} \sqrt{10+10 \alpha_{x}-10 \alpha_{y}}}$. For values of $\omega$ smaller than $\omega_{*}$ external long axis equilibrium points will always exist. As the circumscribing sphere is the spherical boundary where the internal and external potential are matched, the long axis equilibrium points will always be computed with the external potential.

Studying the linearised equations at the equilibria using the external potential it is possible to see that the long axis equilibrium points outside the body always have a saddle-centre behaviour, and are therefore unstable, [10].

\section{Short axis equilibrium point}

Relative equilibrium points aligned with the $y$-axis, called short axis equilibrium points, external to the body can exist for the external ([10]) or for the internal potential depending on the shape and rotation of the body. For the external potential, the equation of the equilibrium points is

$$
\omega^{2}=\frac{1}{y_{e q}^{3}}\left[1+\frac{3}{2 y_{e q}^{2}}\left(1+\alpha_{x}-2 \alpha_{y}\right)\right]
$$

In order to use the external potential the closest equilibrium point will be at the circumscribing sphere, where

$$
\omega=1 / 5 \sqrt{2} \sqrt{-\frac{-16+2 \alpha_{y}+4 \alpha_{x}}{\left(-1-\alpha_{y}+\alpha_{x}\right)^{2} \sqrt{10+10 \alpha_{y}-10 \alpha_{x}}}} .
$$

Analysing the linearised system at the equilibrium it is possible to conclude that the external potential short axis equilibrium point undergoes a Hamiltonian-Hopf bifurcation, which means that it is complex unstable when close to the surface and stable when far from it [10]. Using Scheeres nomenclature, the asteroids with short axis equilibria complex unstable are of type I and when 
the equilibria are stable, of type II. Due to the fact that the density computed by the internal potential vanishes at the circumscribing sphere the Hopf-bifurcation can cross the spherical boundary continuously.

When the angular velocity is smaller than the expression (32) for a given shape, the short axis equilibrium will fall in the area of the internal potential, i.e. $\sqrt{\frac{5}{2}\left(1+\alpha_{x}-\alpha_{y}\right)} \leq y_{e q} \leq$ $\sqrt{\frac{5}{2}\left(1+\alpha_{y}-\alpha_{x}\right)}$. Different choices of the coefficients of the internal potential can slightly vary the boundaries between regions, but the overall behaviour will be the same.

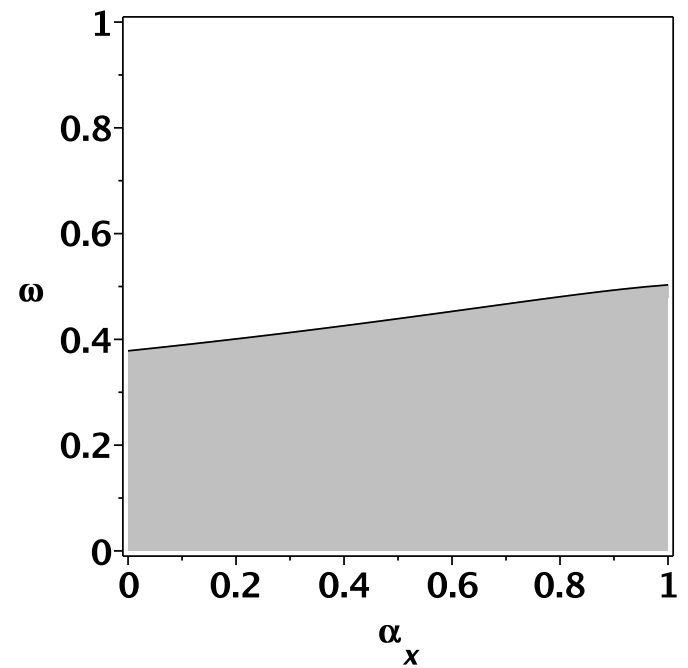

(a) Gray region: long axis equilibrium point is outside the body

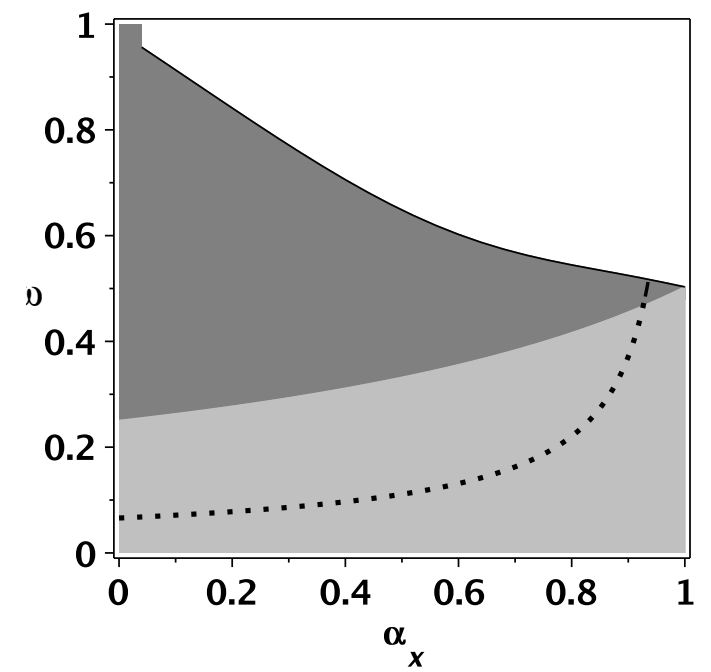

(b) Light gray region: the short axis equilibrium point is computed with the external potential. Dark gray region: it is outside the body but computed with the internal potential. The dotted line corresponds to the Hamiltonian-Hopf bifurcation, below it the short axis equilibrium point is stable.

Fig. 3 Regions in parameter space for the equilibrium points to be computed with external or internal potential and for them to be inside or outside the body when $\alpha_{y}=1$.

This paper is focused on the long axis equilibrium point as the saddle-centre behaviour will guarantee the existence of unstable periodic orbits about the equilibrium with stable and unstable invariant manifolds that might come from or arrive to the asteroid. 


\section{B. Invariant manifolds for the long axis equilibrium}

As the long axis equilibrium is always a saddle-centre, and the periodic orbits close to it will inherit the same instability, stable and unstable manifolds can be computed for both the equilibrium and the nearby periodic orbits [11]. These are manifolds that asymptotically approach, or depart from, the equilibrium point or periodic orbit, giving a handle of how the dynamics behave close to the equilibrium or periodic orbit. This behaviour depends on the rotation period of the central body and its shape.

This section is focused on finding for which shapes and rotations the invariant manifolds approach or intersect the body and how many rotations about the body the manifolds perform before intersecting it or escaping from it. These results can be applied to the design of landing trajectories on asteroids or comets as a spacecraft following an invariant manifold will be able to approach the body with very low relative velocity, orbit around it while observing it, and finally land with a very small increment of velocity.

In order to classify the behaviour of manifolds that intersect the body, the trajectories will be plotted using the angles $\phi$ and $\alpha$ where $\phi$ is the position angle of the trajectory at the intersecting point on the equator of the ellipsoidal surface $(\phi=0$ is aligned with long axis) and $\alpha$ is the angle of the velocity with respect to the tangent to the ellipse at angle $\phi$ ( $\alpha=0$ is against the direction of rotation), see figure 4 .

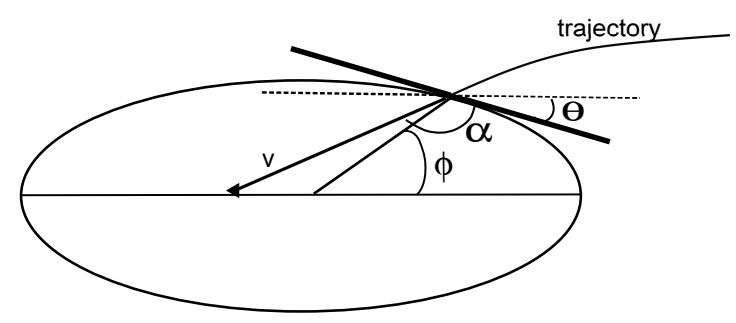

Fig. 4 Angles $\phi$ and $\alpha$ on the equator of the ellipsoid. Angle $\phi$ is the position on the ellipse of the intersecting point of the trajectory. Angle $\alpha$ is the angle between the velocity of the trajectory and the tangent plane at angle $\phi$. The $\theta$ angle is the angle between the tangent plane and the local horizontal. 


\section{Invariant manifolds of equilibrium points}

Given a body with shape $\alpha_{x}$ and $\alpha_{y}$ and fixed angular velocity $\omega$, the energy of the system, the Jacobi constant, $\mathcal{C}$, acts as a parameter. The zero velocity curves given by the Jacobi constant divide the $x, y$ plane in different regions depending on the value of $\mathcal{C},[8,10,11]$. For very small energies, the trajectories are confined in an area next to the body, the interior realm, or they cannot reach the surface of the body, the exterior realm. The separation between the realms is due to an intervening forbidden region where the velocities would be complex.

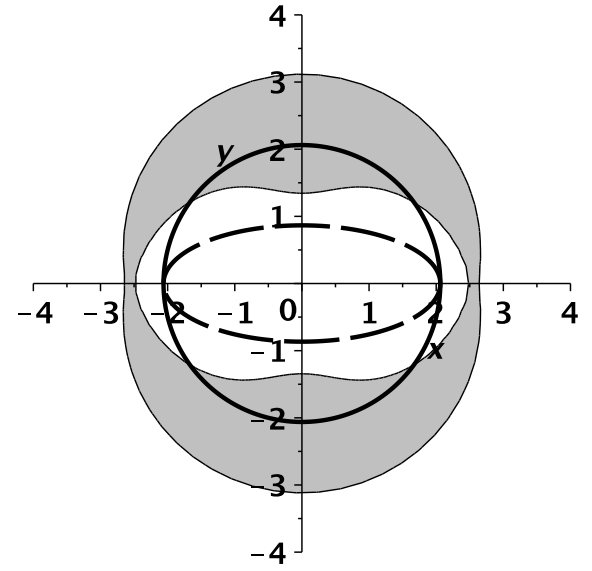

(a) $\mathcal{C}=-0.69$

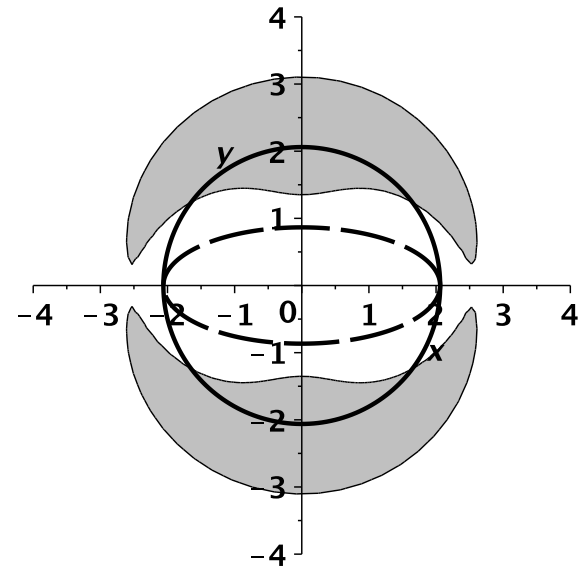

(b) $\mathcal{C}=-0.688$

Fig. 5 Zero velocity curves for a body with $\alpha_{x}=0.3, \alpha_{y}=1$ and $T=6 h$.

As the energy increases, the zero velocity curves open up at the long axis equilibrium point and the exterior realm and interior realm are connected for the first time. At these energies a trajectory coming from far away from the body can approach its surface with low relative velocities compared to trajectories with energies that have the neck region larger and the forbidden areas smaller. Consequently, the invariant manifolds of the long axis equilibrium point define fuel-free paths that approach the asteroid and may be used for landing on it. In [11] the invariant manifolds of the 1:1 synchronous orbit or equilibrium point are used to transfer from higher altitude orbits to lower altitude for Vesta, but without approaching its surface.

In the following simulations, the shape parameter $\alpha_{y}$ is fixed to 1 for simplicity, which means that $I_{y y}=I_{z z}$. This approximately occurs for many asteroids. For different $\alpha_{x} \in(0,1)$ and different periods of rotation, the trajectory that follows the unstable manifold of the respective long 
axis equilibrium point and is directed towards the body is integrated until it impacts on the surface of the ellipse with that value of $\alpha_{x}$, or until a maximum time is reached. In figure 6 the angle $\phi$ on the ellipse of impact as a function of the shape parameter $\alpha_{x}$ is plotted. In this figure only the values $\alpha_{x}$ that put the long axis equilibrium point outside the body are considered.
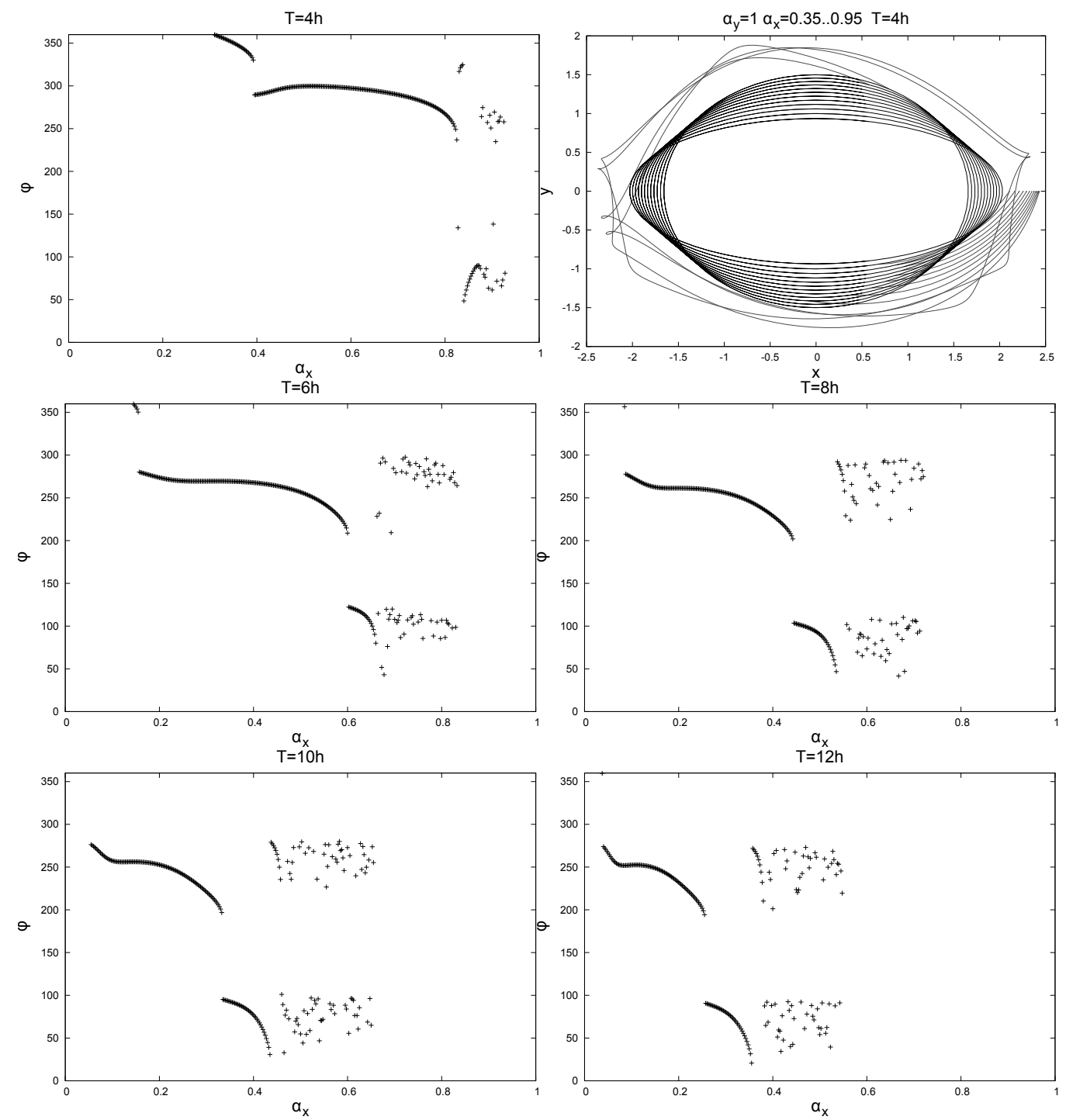

Fig. 6 Impact on the ellipsoid of the unstable manifold of the long axis equilibrium point for rotation periods $T=4,6,8,10$ and 12 hours for $\alpha_{y}=1$ and different shape parameters $\alpha_{x} \in(0,1)$ when the equilibrium point is outside the body. When the impact angle $\Phi$ is 360 means that the equilibrium point is on the surface of the ellipsoid. The upper most right picture represents some of the trajectories until they impact onto the ellipsoid for a rotation period of 4 hours. 
Figure 6 says that as the body becomes more spherical ( $\alpha_{x}$ approaches 1$)$ the unstable manifolds of the long axis equilibrium point start orbiting the body for longer times, until they do not intersect the ellipsoid before the maximum time of integration (for large values of $\alpha_{x}$ no points are plotted). As the rotation period of the body increases the allowable region of $\alpha_{x}$ for impact trajectories decreases. It is possible to observe as well, that the trajectories accumulate in two bands of angle $\phi$ which do not include the long axis region. Since the dynamics are continuous, it means that only for a very small region of $\alpha_{x}$ values the manifold will impact on the long axis Therefore, for the majority of asteroids, a spacecraft following one of these paths without manoeuvring will not be able to land near the long axis of the ellipsoid.

If these manifolds are to be used for landing, it is necessary to know the angle of the velocity at arrival with respect to the surface. Ideally it would arrive vertically, $\alpha=90$ degrees. From figure 7 it is possible to see that the manifolds impact with very large angles, even close to tangentially, which makes these trajectories not suitable without performing a manoeuvre, as the spacecraft may touch the ground, bounce and start orbiting again.

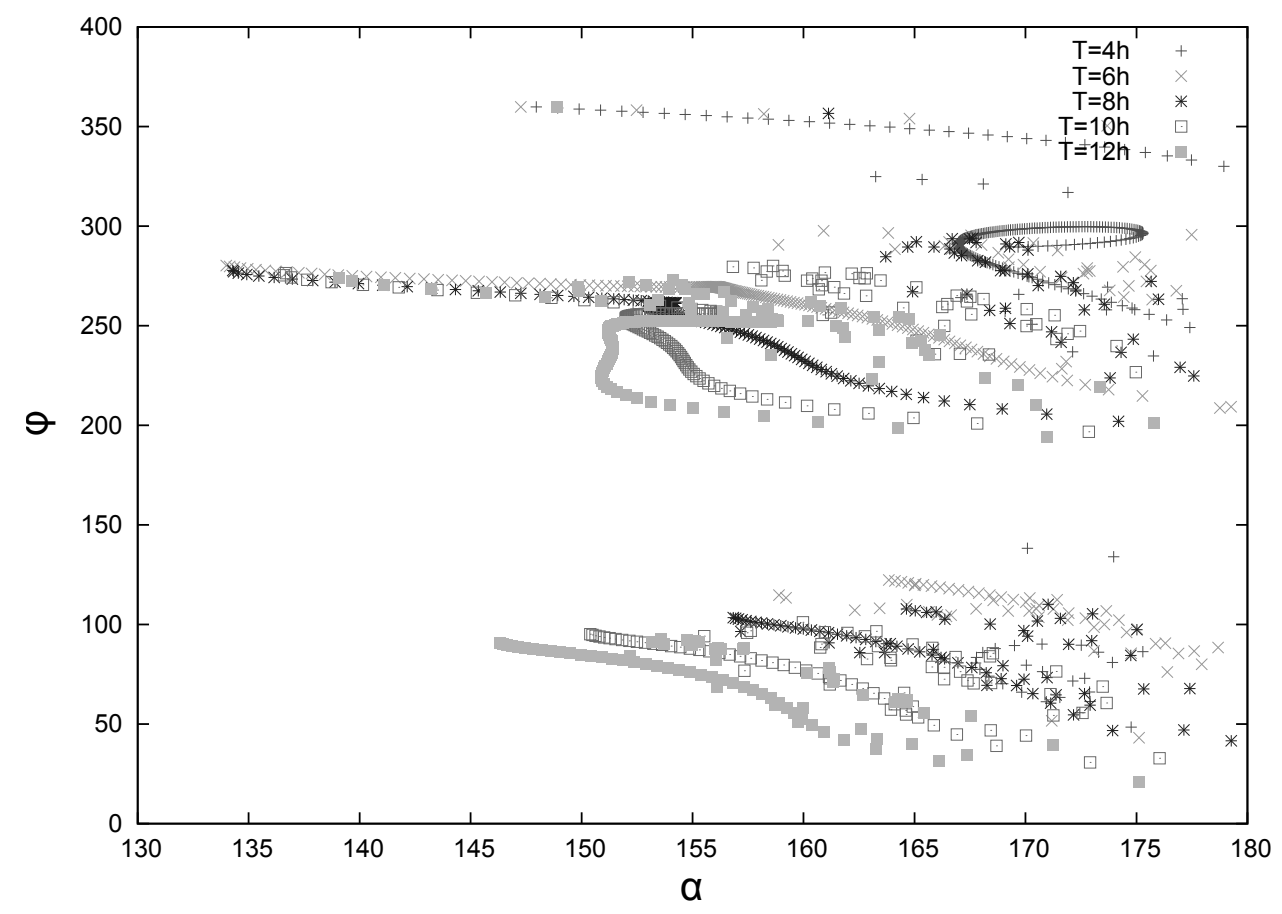

Fig. 7 Angle of arrival $\phi$ and angle of the velocity with respect to tangent, of the different manifolds of figure 6. The different curves of the same symbol represent different shape parameters $\alpha_{x}$. 


\section{Invariant manifolds of periodic orbits}

Periodic orbits about the long axis equilibrium point emanate from it, and so, the invariant manifold of the equilibrium point is the limit of the invariant manifolds of the periodic orbits as the orbits shrink to the equilibrium. By using periodic orbits instead of the equilibrium point, larger approach velocities are allowed, but at the same time other possible angles of the arrival velocity can be found.

In the different plots shown in figure 8 it can be seen that even increasing the energy of the periodic orbits, the manifolds do not seem to intersect the body vertically. In section IV a controlled manoeuvre is proposed to achieve vertical landing. Moreover, observing figure 8 , one can see that by increasing the Jacobi constant of the orbits, a more chaotic behaviour of the manifold can be observed. A study of the behaviour of the manifolds depending on the energy of the orbit and the starting position on the orbit is necessary.

For different shapes and rotations of the elongated body, a family of 150 different periodic orbits about the long axis equilibrium point parameterised by the Jacobi constant were computed. For each orbit, 150 different points with a constant separation in time were selected to compute the stable and unstable manifolds of each periodic orbit. These $150 \times 150$ initial conditions of the unstable manifolds were then followed until they impact on the surface, escape the vicinity of the asteroid or 50 rotations of the central body were completed. The vicinity of the asteroid has been defined as the spherical area centred at the origin with radius the position of the long axis equilibrium point plus the amplitude of the orbit. The first initial condition for the manifold is located on the $x$ axis, on the right side of the orbit. The order of the orbit's quadrants explored is therefore $4,3,2,1$.

For each trajectory, its behaviour and the number of times it went around the body are plotted. Figures 9, 10 and 11 show that as the body becomes spherical, and as the rotation period increases, fewer trajectories impact on the body and more keep orbiting in its vicinity. Moreover, for more spherical bodies and longer rotation periods, the trajectories orbit around the elongated body a higher number of times, allowing for a longer period of observation before landing. Rotation periods higher than 10-12 hours allow the invariant manifolds to circulate around the body several times before impact. 

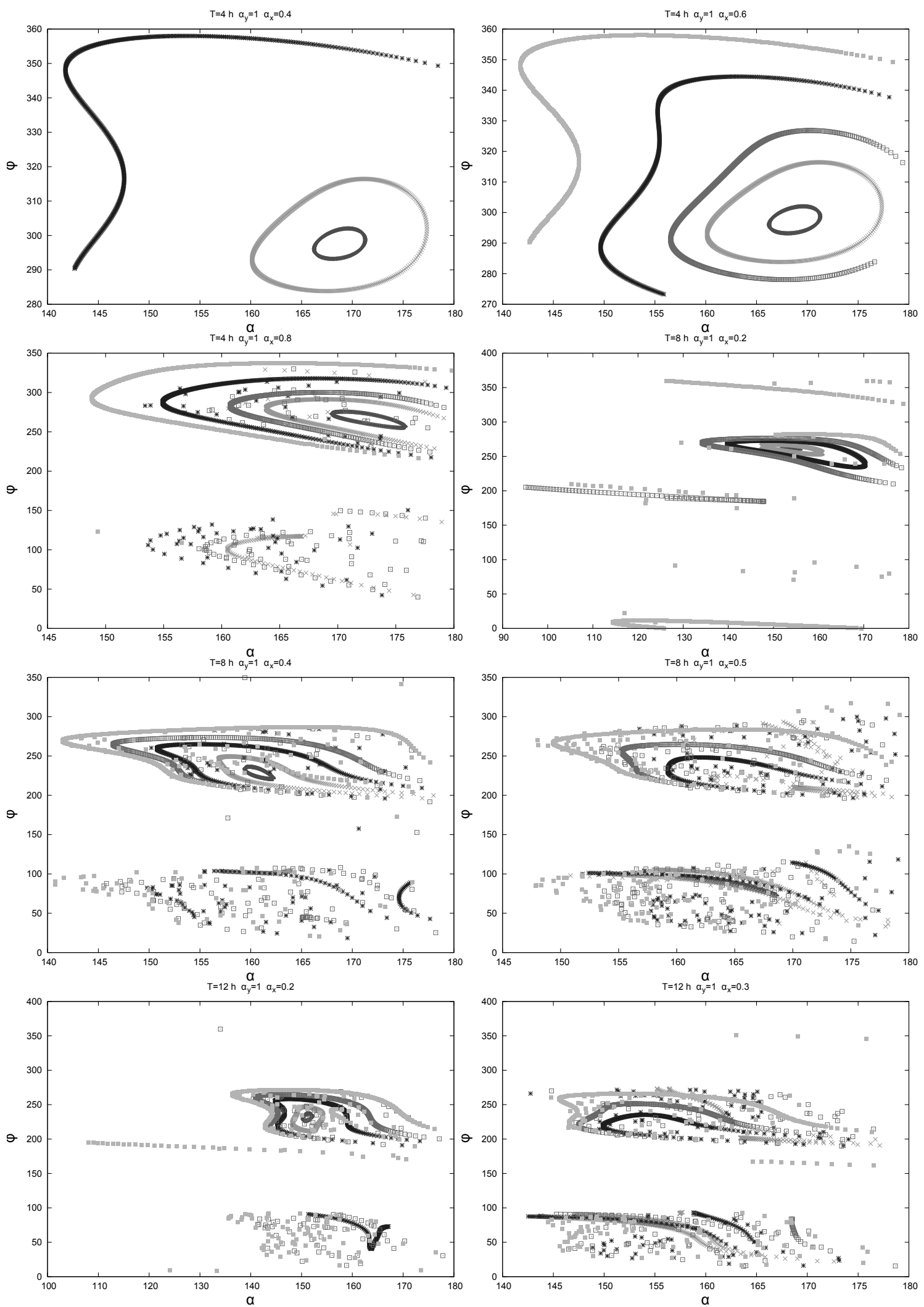

Fig. 8 Impact on the ellipsoid of the invariant manifolds of different periodic orbits, for rotation periods $T=4,8$ and 12 hours, with $\alpha_{y}=1$ and different shape parameters $\alpha_{x} \in(0,1)$. 


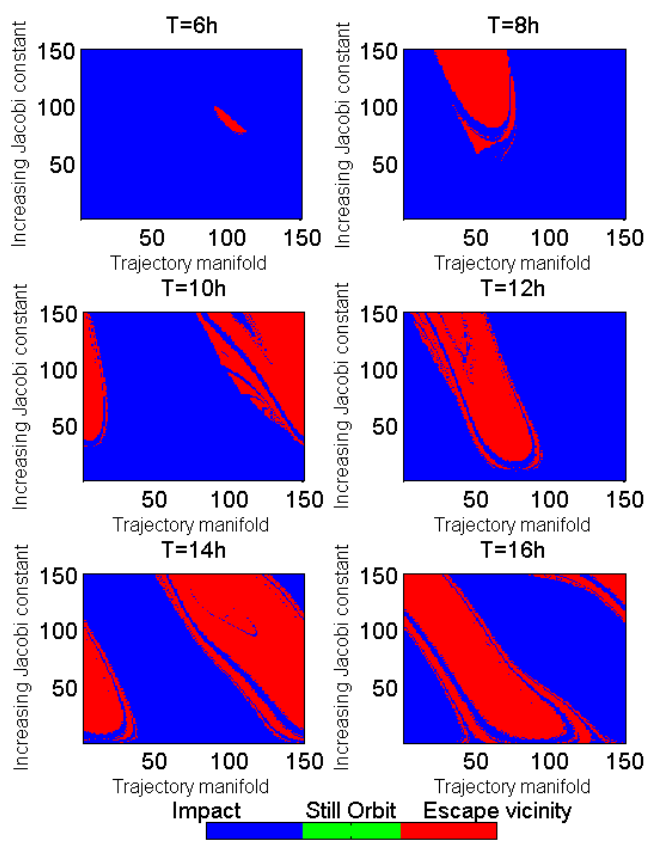

(a) $\alpha_{x}=0.3$
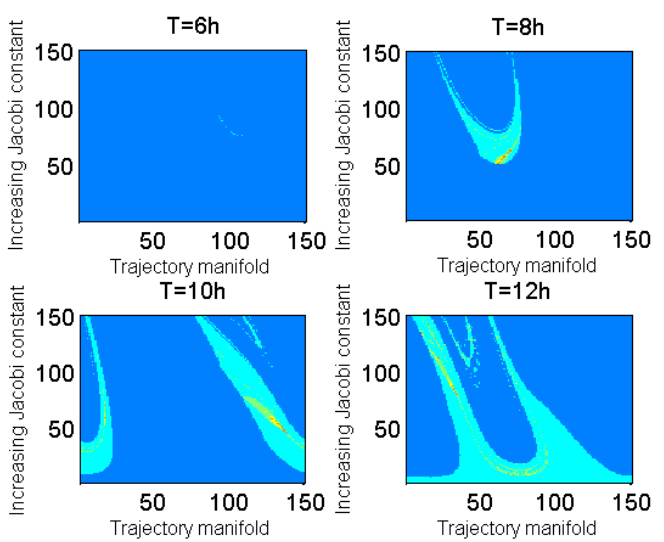

$\mathrm{T}=12 \mathrm{~h}$

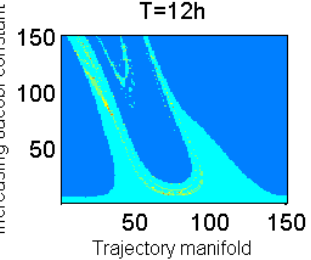

$\mathrm{T}=16 \mathrm{~h}$

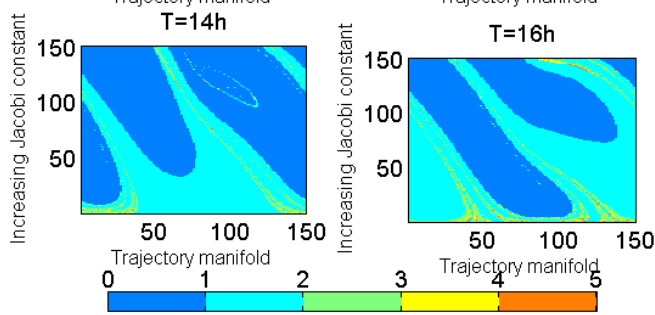

(b) $\alpha_{x}=0.3$

Fig. 9 Left column: behaviour of the unstable manifold trajectories as a function of the Jacobi constant and the position on the orbit, for shape parameter $\alpha_{x}=0.3$ and different rotation periods. The first trajectory on the periodic orbit is on the $x$-axis with $\dot{y}$ negative. The following ones are uniformly distributed in time along the periodic orbit and therefore belong to the 4th, 3rd, 2 nd and 1st quadrants respectively. Right column: number of rotations about the elongated body that the unstable manifold trajectories perform during 50 rotations of the body. The number of rotations is given by the colorbar: from 0 to 1 , from 1 to 2 , etc., until more than 5 rotations.

Observing figures 9, 10 and 11 it is possible to see large regions where the manifolds escape the vicinity of the asteroid. Although the trajectories integrated correspond to the invariant manifold directed to the non-spherical body, many of them, depending on the shape and rotation, after approaching the asteroid they leave its vicinity through the gap of the zero velocity curves at any of the long axis equilibrium points.

IV. Possibility of landing on asteroids using invariant manifolds

There have been two missions that included controlled landings on asteroids: the NEAR mission to asteroid Eros [29] and the Hayabusa mission to asteroid Itokawa [6]. However, neither of these 

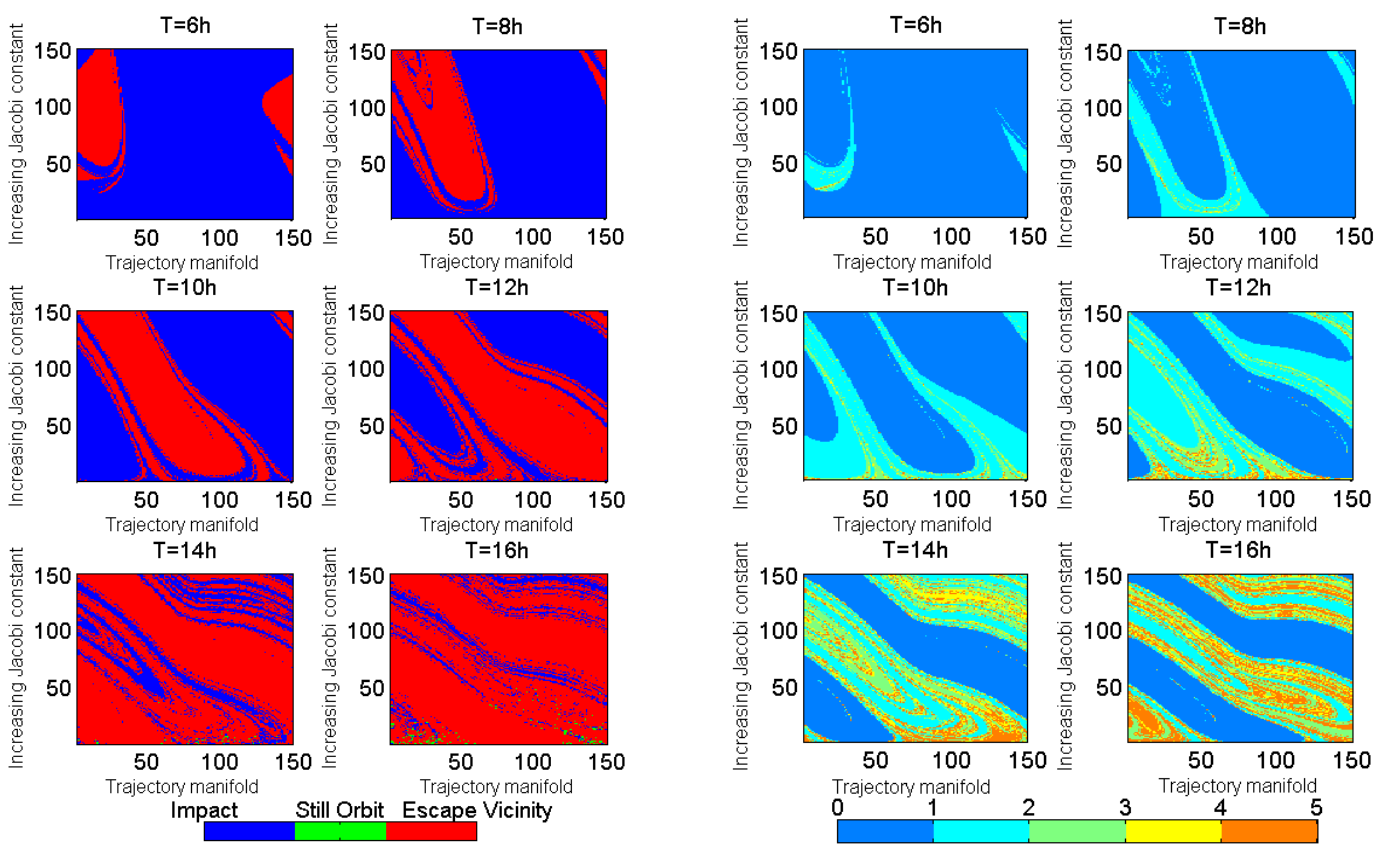

(a) $\alpha_{x}=0.5$

(b) $\alpha_{x}=0.5$

Fig. 10 Same as figure 9 but with $\alpha_{x}=0.5$.
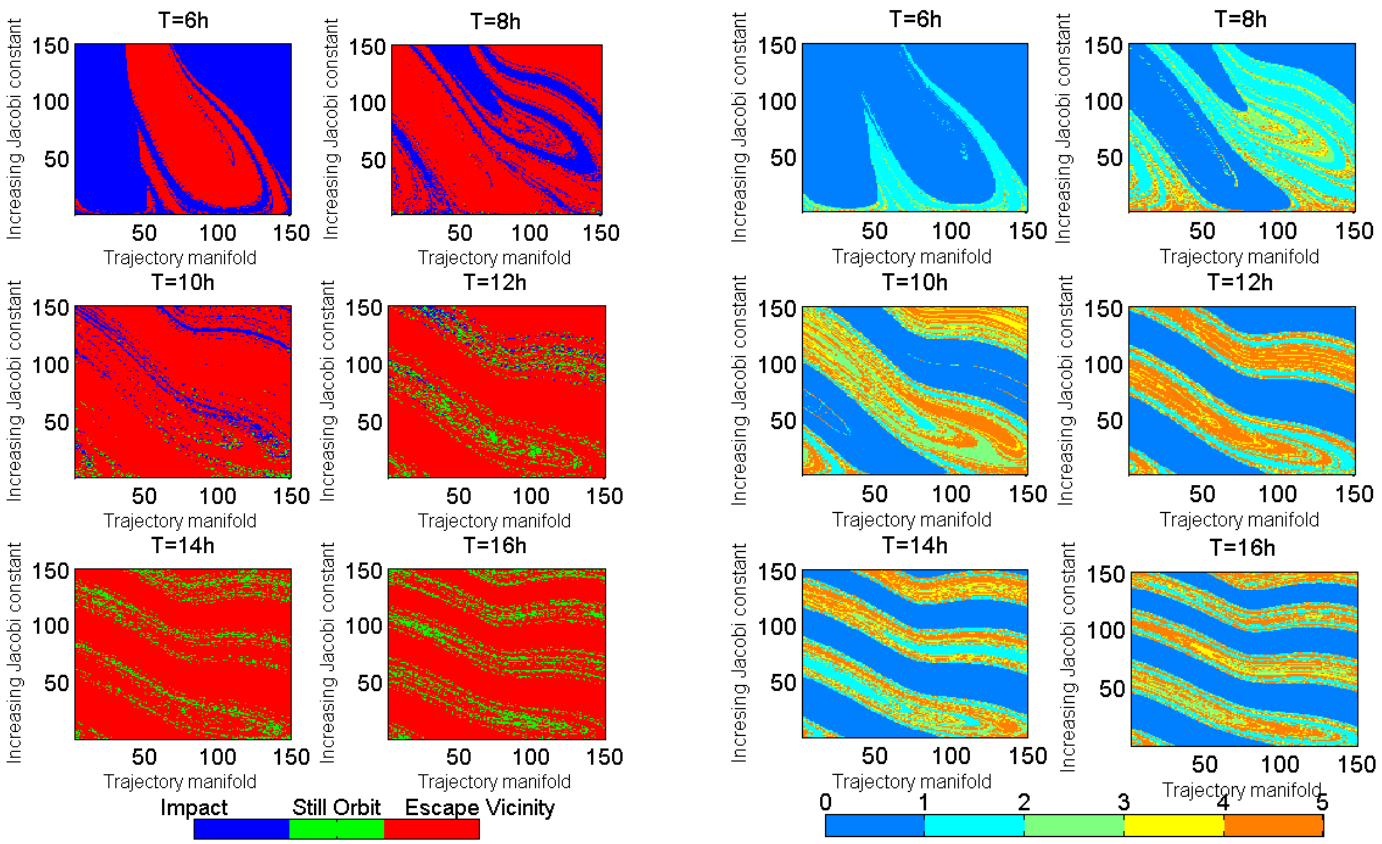

(a) $\alpha_{x}=0.7$

(b) $\alpha_{x}=0.7$

Fig. 11 Same as figures 9 and 10 but with $\alpha_{x}=0.7$.

missions used the natural dynamics about the Lagrange points as part of the landing manoeuvres, even though good approximations to the shape and gravitational potential of the model were com- 
puted by the same spacecraft while orbiting the asteroid. In this section a methodology for close observation of the asteroid and later landing that takes advantage of the invariant manifolds of periodic orbits close to the surface is proposed.

In previous sections it has been seen that the unstable manifolds of periodic orbits can provide fuel-optimal trajectories that approach and intersect the body with small relative velocities. In a similar way, trajectories from the stable manifold come from far away from the body and approach the periodic orbit. Therefore, by adding small manoeuvres to appropriate trajectories from the stable and unstable manifolds a close rendezvous trajectory and soft landing can be achieved.

Given a periodic orbit around the long axis equilibrium point, a trajectory of the stable manifold can take a spacecraft from the exterior realm to the periodic orbit without using fuel. Therefore, the first manoeuvre needed is the insertion onto the appropriate stable manifold. Once the spacecraft is on the desired periodic orbit, a jump to the unstable manifold is required. This is the second manoeuvre. Then the spacecraft will travel on the unstable manifold without thrusting, approaching the body in the interior realm. Finally, once the spacecraft has orbited the body and decided where to land, the last manoeuvre, a vertical landing, has to be performed.

In this section it is shown how the landing can be performed using invariant manifolds. An example with a real asteroid (Nereus) is given with the required increments of velocity computed for each trajectory. The aim is to use a trajectory that starts on a parking orbit around the asteroid in the exterior realm, approaches it via the stable manifold of a particular Lyapunov periodic orbit around the Lagrange point, and then orbits the asteroid several times in the interior realm on the unstable manifold, before performing an orthogonal landing.

Asteroid 4660 Nereus is a small Apollo and Mars crosser of dimensions $2 a=510 \mathrm{~m}, 2 b=330 \mathrm{~m}$ and $2 c=241 \mathrm{~m}$. Its orbit approaches the Earth frequently which makes this asteroid very accessible to spacecraft missions due to the low $\Delta v$ needed for rendezvous. Moreover, the rotation period of this asteroid is $T=15.16$ hours, which makes it very suitable for the theory developed in this paper. The shape parameters for asteroid Nereus are $\alpha_{x}=0.4525$ and $\alpha_{y}=0.8623$. In order to compute an approximate mass a mean density of $2 \mathrm{~g} / \mathrm{cm}^{3}$ has been assumed.

In order to design the whole trajectory, it is necessary to first select the desired Lyapunov orbit 
around the long axis Lagrange point, as the whole trajectory depends on it. This orbit should have an unstable manifold that approaches the asteroid and orbits it several times at low relative velocity. Thus, the analysis of the behaviour of the unstable manifolds of different periodic orbits around the long axis Lagrange point as in figure 9-11 is the first step. 200 orbits were selected, and for each orbit, 200 trajectories were followed until they intersected the body, escaped from the vicinity or a maximum time was reached. This maximum integration time was 50 rotations of Nereus.

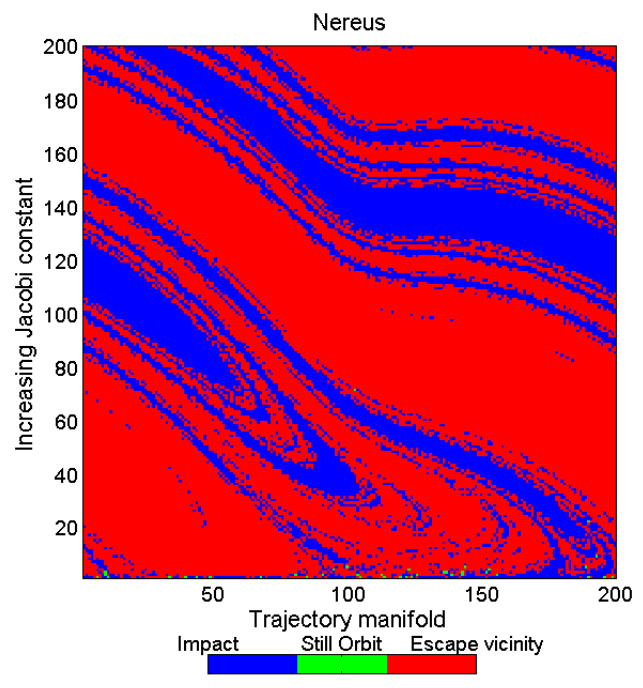

(a) Behaviour manifolds

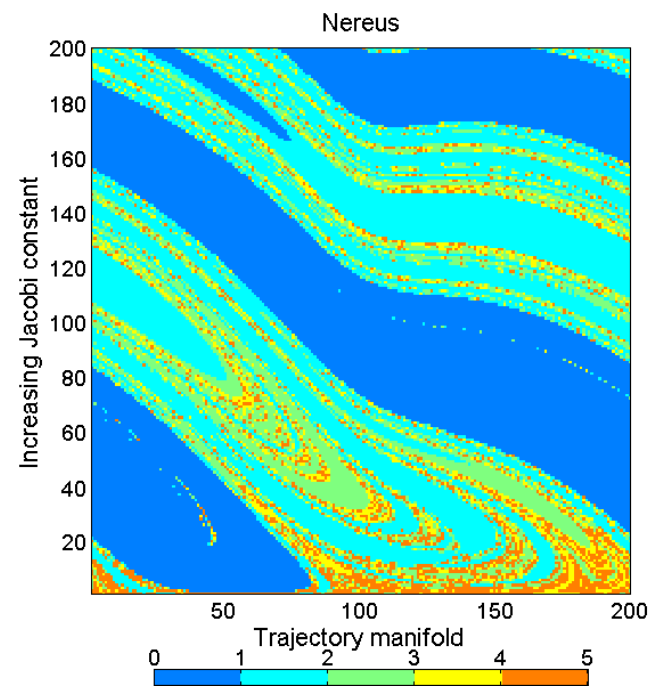

(b) Number of rotations around body

Fig. 12 Behaviour of 200 different trajectories for each of the 200 different periodic orbits about the long axis equilibrium point of asteroid Nereus.

Observing figure 12 it is possible to see that for small periodic orbits (lower part of the graph on the right hand side) the trajectories go around the asteroid Nereus a larger number of times. Therefore, for a mission that first wants to observe and study the asteroid for some time and later land on a desired spot, this kind of periodic orbit seems suitable. For this example of a complete landing manoeuvre, the small periodic orbit around the Lagrange point with initial conditions

$$
\begin{aligned}
& x=4.878060190404, \\
& y=0.0, \\
& \dot{x}=0.0, \\
& \dot{y}=-0.1253951750843,
\end{aligned}
$$


and Jacobi constant $\mathcal{C}=-0.3572213504562$ plotted in figure 13 has been selected. This figure also shows two stable retrograde orbits around the asteroid that can be used as the initial parking orbit. The parking orbit should be stable as the spacecraft will stay on it for a period of time in order to study the asteroid and compute the gravitational potential. As the final aim of the mission is to land on the spacecraft using invariant manifolds, the parking orbit should be chosen in such a way that the trajectories from the stable manifold of the desired Lyapunov periodic orbit intersect the parking orbit.

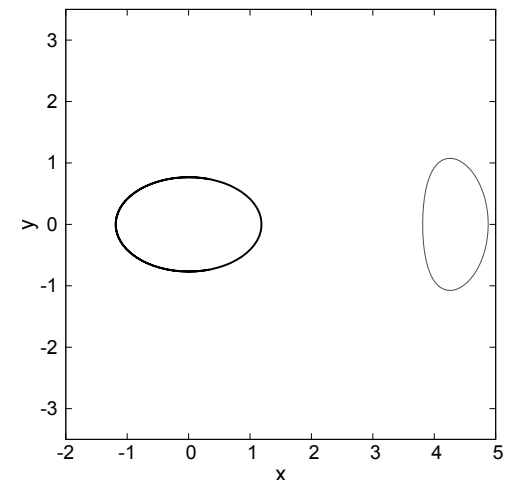

(a) Lyapunov periodic orbit around Lagrange point with $\mathcal{C}=-0.3572213504562$

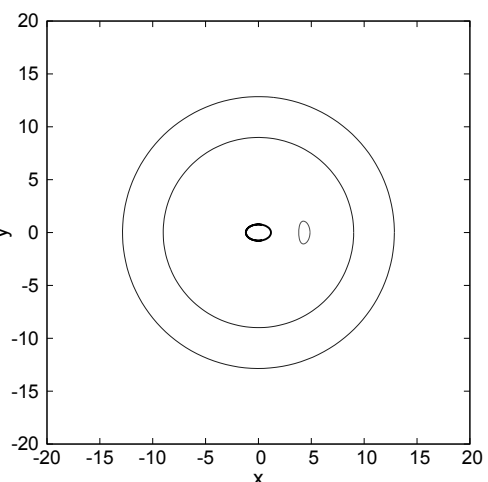

(b) Stable periodic orbits around Nereus that can be used as parking orbits

Fig. 13 Periodic orbits selected for the mission. The asteroid Nereus is plotted in thick black line.

\section{A. Manoeuvre 1: insertion onto the stable manifold}

It is assumed that at the beginning of the landing manoeuvre, the spacecraft is already in a stable periodic orbit around the asteroid in rotating coordinates. The radius of the orbit will be between 10 to 20 non-dimensional units, which is between approximately 5 and 10 radii of the system, as plotted in figure 13 .

The stable manifold trajectories that come from the exterior realm are retrograde in the rotating frame and circulate around the body many times, some of them reaching back to the stable parking orbit. The first manoeuvre is performed at an intersection point between the manifold and the orbit. In order to find an intersection point, different trajectories on the stable manifold of the selected 
periodic orbit are integrated backwards.

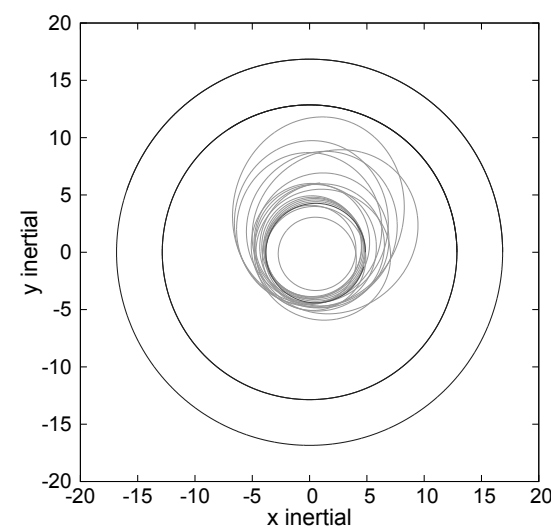

(a)

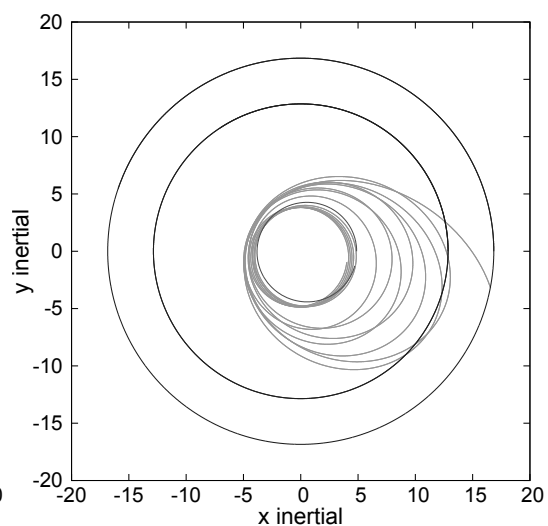

(b)

Fig. 14 Two different trajectories from the stable manifold of the periodic orbit (plotted in light gray) and two stable orbits about the asteroid in inertial coordinates. The right trajectory intersects the orbits about the asteroid.

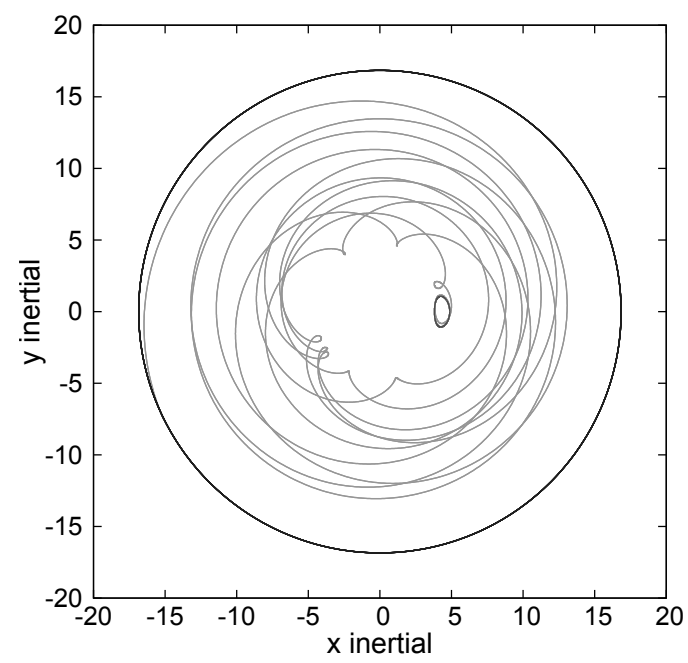

Fig. 15 Intersection of the stable manifold trajectory and the largest parking orbit considered in rotating coordinates.

The choice of the stable manifold trajectory and the point at which the insertion manoeuvre onto the manifold is performed will depend on the choice of initial parking orbit and the desired time of transfer. In principle a detailed study should explore the trade-off between the time taken to transfer and the $\Delta v$, but this goes beyond the scope of this paper where the focus on the last part of the landing manoeuvre. Therefore a particular orbit and trajectory of the manifold to compute 
the required manoeuvres has been selected. Other choices of orbits with lower $\Delta v$ are possible. Due to the fact that not all the Lyapunov orbits around the long axis equilibrium point have to have stable manifolds that intersect the parking orbit, the choice of the parking orbit will depend on the initial choice of the Lyapunov orbit.

Having selected as the initial parking orbit the one in figure 15 with initial conditions in nondimensional units

$$
\begin{aligned}
& x=16.8456946730,2 \\
& y=0.0, \\
& \dot{x}=0.0, \\
& \dot{y}=-1.684873324352
\end{aligned}
$$

and period $T=62.82228500699$, and the manifold trajectory shown in figure 15 , with initial conditions

$$
\begin{aligned}
& x=4.018802203493, \\
& y=-0.9477276602606, \\
& \dot{x}=-0.04843510212615, \\
& \dot{y}=0.06122417274927,
\end{aligned}
$$

if a manoeuvre is performed at the point $x=-15.247829, y=-7.160792$ in rotating coordinates which corresponds to non-dimensional time $t=-1295.347146078$ we have the following nondimensional inertial velocities:

$$
\begin{aligned}
\dot{x}_{\text {orbit }} & =-0.716254, \\
\dot{y}_{\text {orbit }} & =1.525018, \\
\dot{x}_{\text {manifold }} & =-0.666349, \\
\dot{y}_{\text {manifold }} & =1.637142 .
\end{aligned}
$$

Then, the $\Delta v$ for this manoeuvre is 0.122723 in non-dimensional units which corresponds to $1.3048968 \cdot 10^{-4} \mathrm{~s}^{-1}$. As the fundamental unit of length for asteroid Nereus is $R=135.83 \mathrm{~m}$, 
the $\Delta v$ for the first manoeuvre is $0.01772 \mathrm{~ms}^{-1}$. The time of transfer for this manoeuvre is approximately 14.1 days.

\section{B. Manoeuvre 2: jump to the unstable manifold}

Once the trajectory has approximately reached the periodic orbit, a very small manoeuvre has to be done to perturb the spacecraft in the direction of the unstable manifold to follow a desired unstable trajectory. The branch of the unstable manifold chosen is the one that departs from the orbit in the direction of the interior realm.

Looking in detail at figure 12 it is possible to see that the initial points on the orbit that perform more number of turns before impacting or escaping fall on the right hand side of the plot. This means that the suitable starting points for the unstable manifold are approximately on the second and first quadrant of the periodic orbit, where the 0 angle is defined as the intersection with larger $x$ between the periodic orbit and the $x$-axis. The $\Delta v$ to perform the jump is negligible as the stable and unstable manifold have the same Jacobi constant.

\section{Manoeuvre 3: vertical descent}

When the spacecraft is on the unstable manifold it will approach the surface of the asteroid without any thrusting. The trajectory selected for the landing manoeuvre is plotted in figure 16 and it has the following initial conditions

$$
\begin{aligned}
& x=4.518027515857, \\
& y=0.9608006701563, \\
& \dot{x}=0.06475124539802, \\
& \dot{y}=-0.05660351029247 .
\end{aligned}
$$

This orbit has been selected because it goes around the asteroid 4 times before impacting on the surface. Then, the spacecraft has time to observe the asteroid at a safe distance before deciding where to land.

Figure 16 shows that if a manoeuvre is not performed, the trajectory will impact the asteroid with a very tangential velocity. Therefore, it is possible that depending on the coefficient of restitu- 


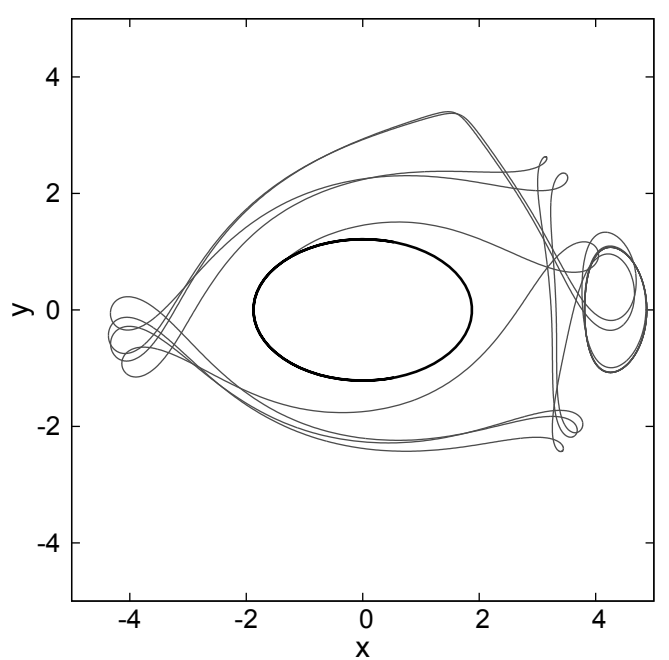

Fig. 16 Trajectory of the unstable manifold for the periodic orbit selected. The trajectory starts on the first quadrant of the periodic orbit.

tion of the asteroid, instead of remaining on the surface of the asteroid the spacecraft might go into orbit again. In order to have a safe landing on an asteroid, it is desirable to follow a trajectory which arrives at the surface orthogonally. However, the different manifolds inspected in this paper that intersect asteroid Nereus and other elongated bodies with different shape and rotation parameters, do so with a very large angle, between 140 and 180 degrees, see figure 8 . This is not a suitable descent angle for real missions. Therefore, a control algorithm must be implemented in order to achieve a vertical landing. This control algorithm can also be implemented on manifold trajectories that do not impact on the body and it will guarantee an impact with the surface.

A suitable way of studying this problem is by using orthogonal elliptic coordinates. The orthogonal elliptic coordinates lines are confocal ellipses and their orthogonal hyperbolae. To define the focal distance of the coordinates the foci of the ellipse that has the same $\alpha$ parameters as the equator of the asteroid considered are used. Therefore, the foci are located at $(-f, 0)$ and $(f, 0)$ where $f=\sqrt{a^{2}-b^{2}}$ and $a=\sqrt{5 / 2\left(1+\alpha_{y}-\alpha_{x}\right)}$ and $b=\sqrt{5 / 2\left(1+\alpha_{x}-\alpha_{y}\right)}$ are the normalised semi-major and semi-minor axes of the ellipse. Therefore, the equatorial ellipse of the body defines the coordinates.

The elliptic coordinates $(\mu, \nu)$ are defined by the following transformation to Cartesian coordi- 
nates

$$
\begin{aligned}
& x=f \cosh \mu \cos \nu, \\
& y=f \sinh \mu \sin \nu,
\end{aligned}
$$

where $\mu \in \mathbb{R}^{+}$and $\nu \in[0,2 \pi]$. In these coordinates, trajectories with constant $\mu$ form ellipses and trajectories with constant $\nu$ form hyperbolas. If the landing trajectory follows a hyperbola, the touch down on the elliptical surface will be orthogonal. Therefore, the control algorithm will have to satisfy $\dot{\nu}=0$ to guarantee orthogonal landing. In addition, the control algorithm will have to guide the spacecraft to $\mu=\mu_{0}$ which defines the equatorial ellipse and surface of the body. Due to the fact that the manifold trajectories approach the body with very low relative velocity, the landing manoeuvre does not include a reduction of the velocity as the velocity at touch down will be very small.

The landing manoeuvre will be as follows. First of all, the spacecraft will follow freely a particular unstable manifold around the body while inspecting it. This particular trajectory of the unstable manifold has been selected so that it orbits around the asteroid several times, allowing the spacecraft to have a good observation time. At the moment a suitable spot for landing has been selected, the controller will be turned on. The control law will be a pd controller. At each time step, the trajectory will be at $(x, y, \dot{x} \dot{y})$ which have their corresponding $(\mu, \nu, \dot{\mu}, \dot{\nu})$. It is desired that $\mu$ should approache $\mu_{0}$ and $\dot{\nu}=0$. Therefore, the following errors are defined:

$$
\begin{aligned}
\text { error }_{x} & =f \cosh \mu_{0} \cos \nu-f \cosh \mu \cos \nu, \\
\text { error }_{y} & =f \sinh \mu_{0} \sin \nu-f \sinh \mu \sin \nu, \\
\text { errord }_{x} & =f \dot{\mu} \sinh \mu \cos \nu-f(\dot{\mu} \sinh \mu \cos \nu-\dot{\nu} \cosh \mu \sin \nu), \\
\text { errord }_{y} & =f \dot{\mu} \cosh \mu \sin \nu-f(\dot{\mu} \cosh \mu \sin \nu+\dot{\nu} \sinh \mu \cos \nu) .
\end{aligned}
$$

Note that in the $\operatorname{errord}_{x}$ and errord $_{y}$ expressions the term $\mu_{0}$ does not appear and instead there is the variable $\mu$. It was considered in this application that the proportional part of the controller would take care of the error in position and the derivative part of the controller would take care of the error in velocities. It is possible as well to consider $\operatorname{error} d_{x}=f \dot{\mu} \sinh \mu_{0} \cos \nu-$ $f(\dot{\mu} \sinh \mu \cos \nu-\dot{\nu} \cosh \mu \sin \nu)$ and $\operatorname{errord}_{y}=f \dot{\mu} \cosh \mu_{0} \sin \nu-f(\dot{\mu} \cosh \mu \sin \nu+\dot{\nu} \sinh \mu \cos \nu)$, 
although the results in this paper are computed with the expressions of the errors from equations (35)-(38). Then

$$
\mathbf{u}=\left(u_{1}, u_{2}\right)^{t}=K p \cdot\left(\operatorname{error}_{x}, \text { error }_{y}\right)^{t}+K d \cdot\left(\operatorname{errord}_{x}, \operatorname{errord}_{y}\right)^{t} .
$$

To achieve vertical landing for the trajectory of the unstable manifold for asteroid Nereus plotted in figure 16, a controlled landing following approximately a hyperbola is performed. The starting point of the controlled trajectory can be anywhere on the unstable manifold trajectory, it will only depend on where the spacecraft is desired to land. The resulting controlled trajectory is plotted in figure 17 .

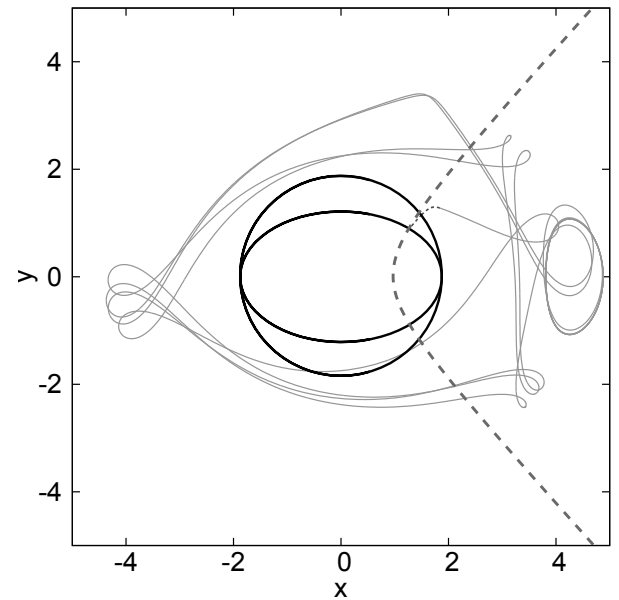

(a) Rectified trajectory for landing on Nereus

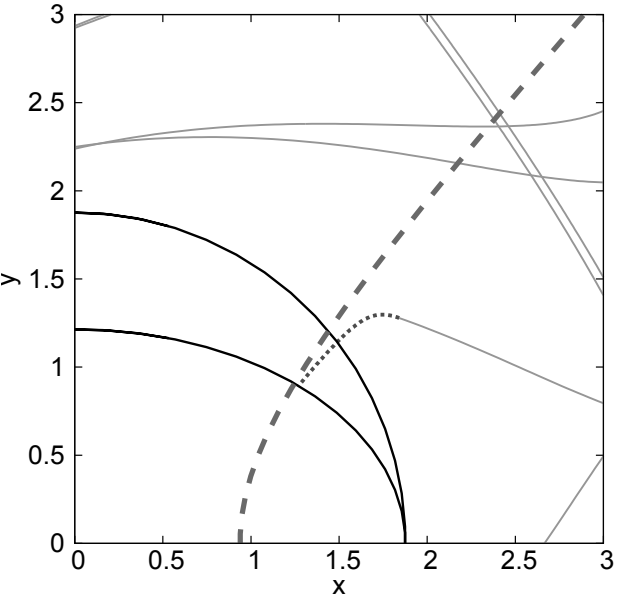

(b) Zoom view of the landing trajectory

Fig. 17 In light gray a particular trajectory of the unstable manifold. The small dashed line represents the corrected landing to achieve orthogonal intersection with the surface of asteroid Nereus. The large dashed line is the orthogonal hyperbola with the same intersecting point. The controller gains for this trajectory are set in non-dimensional units as: $K p=0.05$ and $K d=3.0$.

From the parking orbit, dividing the landing in these three legs: insertion onto the stable manifold, jump to stable manifold and vertical landing, a soft controlled landing manoeuvre has been achieved. The increments of velocity required are summarised in table 1 . Therefore, for the whole landing manoeuvre the total $\Delta v=0.1153 \mathrm{~ms}^{-1}$ and the final velocity on arrival is 0.1097 $\mathrm{ms}^{-1}$. 


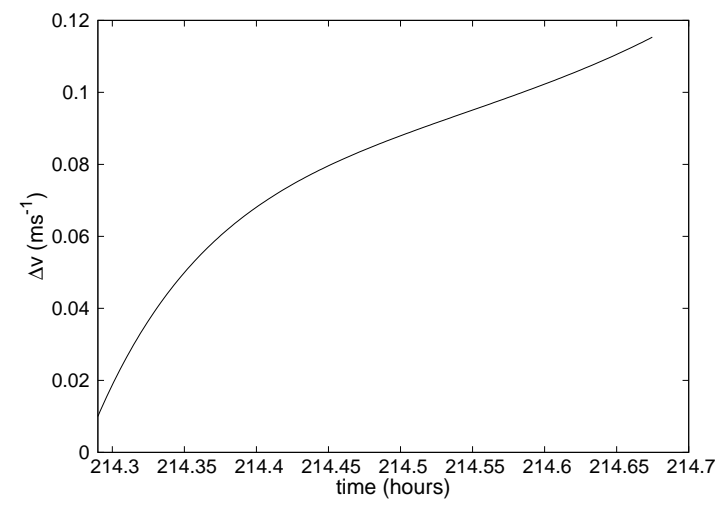

(a) Magnitude of the $\Delta v$ used

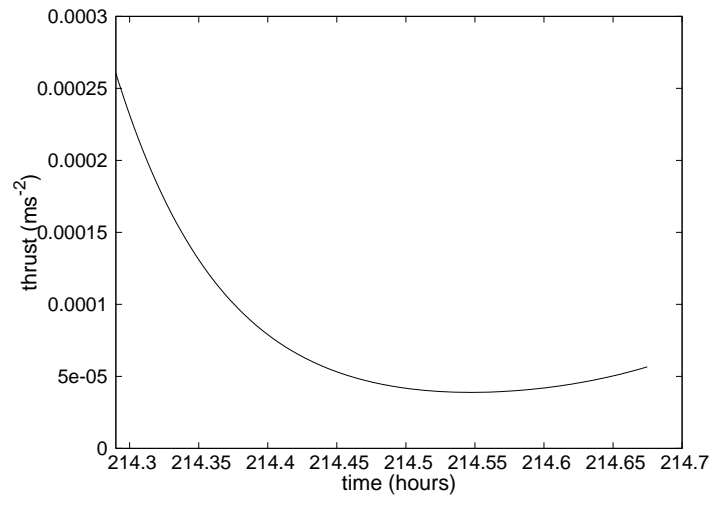

(b) Thrust required to perform the manoeuvre

Fig. 18 Thrust and $\Delta v$ as a function of time for the last part of the landing trajectory.

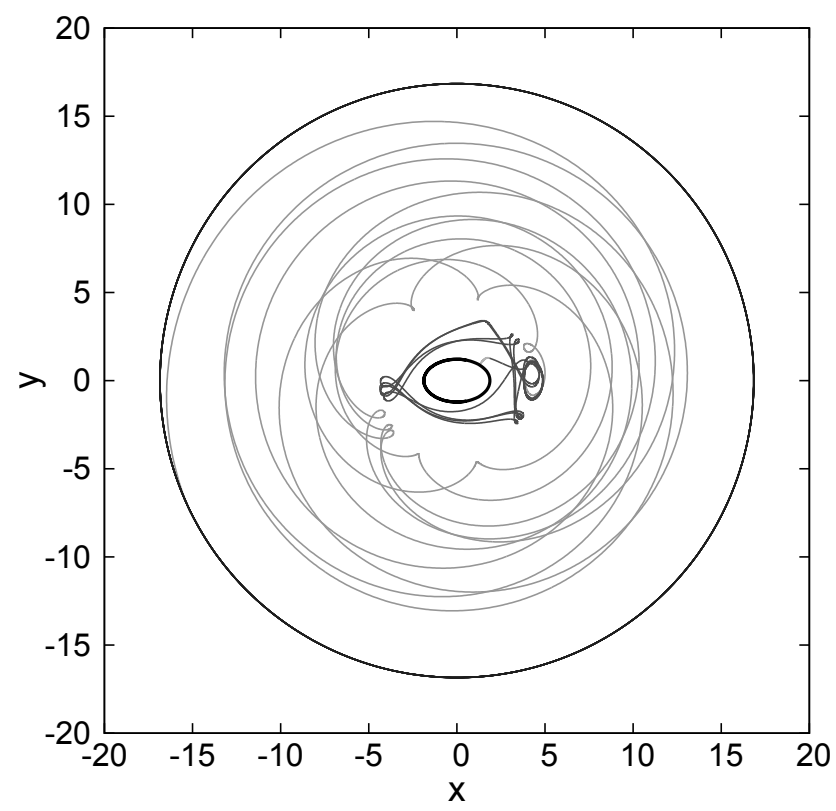

Fig. 19 All the landing manoeuvre from the parking orbit in rotational coordinates.

\begin{tabular}{|l|l|l|}
\hline Leg & $\Delta v$ & Time of flight \\
\hline \hline insertion stable manifold & $0.01772 \mathrm{~ms}^{-1}$ & 14.1 days \\
\hline jump unstable manifold & $\approx 0.0 \mathrm{~ms}^{-1}$ & \\
\hline vertical landing & $0.1132 \mathrm{~ms}^{-1}$ & 8.94 days \\
\hline
\end{tabular}

Table 1 Increments of velocity and time of flight for a landing manoeuvre on asteroid Nereus.

\section{Conclusions}

In this paper, using a gravitational potential only in terms of the mass and moments of inertia, the dynamical environment of elongated bodies of different shapes and different rotation periods has 
been analysed, with a particular focus on the invariant manifolds of periodic orbits around equilibria. It has been seen that, depending on the shape and rotation rate, the invariant manifolds of periodic orbits about the long axis relative equilibrium point can come from or impact onto the surface of the body, providing fuel-efficient trajectories for landing on the surface. We have seen that for fast rotating asteroids that have the long axis equilibrium points outside the body, the manifolds impact quickly on the surface. However, when the rotation period is increased fewer trajectories from the manifold impact on the surface and more of them circulate around the body before later escaping. Increasing the elongation of the asteroid causes more trajectories to quickly impact on the surface, whereas for quasi spherical bodies, the invariant manifolds spend a long time in the vicinity of the body but do not escape or impact on it.

Finally, analysing the behaviour of the invariant manifolds of periodic orbits a methodology that can be used to design a landing mission on an asteroid has been presented. This methodology is valid for elongated asteroids with rotation periods that allow the long axis equilibrium points to be outside of the body, and it is more useful for rotation periods longer than 10-12 hours. Longer rotation periods give the spacecraft more time for observation of the asteroid as the manifolds circulate around the body several times before impact. In order to achieve a vertical landing, and so reduce the possibility of going again into orbit after touch down, a simple control strategy has been designed. With this methodology the landing manoeuvre from a stable quasi-circular parking orbit in the exterior realm but close enough to the asteroid (5-10 radii distance) can be achieved with small increments of velocity of the order $0.15 \mathrm{~ms}^{-1}$ for an asteroid with semi-major axis of 1 $\mathrm{km}$ or $1.5 \mathrm{~ms}^{-1}$ for an asteroid with semi-major axis of $10 \mathrm{~km}$.

\section{Acknowledgements}

The research has been partially funded by the European Commission through the Astrodynamics Network under Marie Curie contract MRTN-CT-2006-035151.

\section{References}

[1] A.F. Cheng. Near Earth Asteroid Rendezvous: Mission Summary. Asteroids III, pages 351-366, 2002. 
[2] C.T. Russell, editor. Deep impact mission: looking beneath the surface of cometary nucleus, volume 117 of Space Science Reviews. Springer, The Netherlands, 2005.

[3] D.E. Brownlee, P.Tsou, J.D. Anderson, M.S. Hanner, R.L. Newburn, Z. Sekanina, B.C. Clark, F.Horz, M.E. Zolensky, J. Kissel, J.A.M. McDonnell, S.A. Sandford, and A.J. Tuzzolino. Stardust: Comet and interstellar dust sample return mission. Journal of Geophysical Research, 108(E10), 2003. doi:10.1029/2003JE002087.

[4] C.T. Russell, F.Capaccioni, A.Coradini, M.C. De Sanctis, W.C. Feldman, R.Jaumann, H.U. Keller, T.B. McCord, L.A. McFadden, S. Mottola, C. M. Pieters, T. H. Prettyman, C. A. Raymond, M.V. Sykes, D.E. Smith, and M.T. Zuber. Dawn mission to Vesta and Ceres. Symbiosis between terrestrial observations and robotic exploration. Earth Moon Planet, (101):65-91, 2007. doi: 10.1007/s11038-0079151-9.

[5] K.H. Glassmeier, H. Boehnhardt, D. Koschny, E. Kührt, and I. Richter. The rosseta mission: Flying towards the origin of the Solar System. Space Science Reviews, (128):1-21, 2007. doi: 10.1007/s11214006-9140-8.

[6] T. Kubota, T. Hashimoto, and J. Kawaguchi. Guidance and navigation of Hayabusa spacecraft for asteroid exploration and sample return mission. Sice-Icase International Joint Conference, October 18-21 2006.

[7] K. Gozdziewski. Nonlinear stability of the lagrangian libration points in the Chermnykh problem. Celestial Mechanics and Dynamical Astronomy, 70:41-58, 1998. 10.1023/A:1008250207046.

[8] B. Chauvineau, P. Farinella, and F. Mignard. Planar orbits about a triaxial body: Application to asteroidal satellites. Icarus, 105:370-384, 1993.

[9] B. Chauvineau, P. Farinella, and F. Mignard. Orbits about a triaxial spinning body. Seventy-five (75) years of Hirayama asteroid families: The role of collisions in the Solar System history. Astronomical Society of the Pacific Conference Series, Vol. 63, Proceedings of the international conference; San Francisco: Astronomical Society of the Pacific (ASP); edited by Yoshihide Kozai, Richard P. Binzel, and Tomohiro Hirayama:45, 1994.

[10] D.J. Scheeres. Dynamics about uniformly rotating triaxial ellipsoids: application to asteroids. Icarus, 110:225-238, 1994.

[11] J.M. Mondelo and S.S. Broschart and B.F.Villac. Dynamical Analysis of 1:1 Resonances near Asteroids: Application to Vesta. AIA A/AAS Astrodynamics Specialist Conference, Toronto, Canada Aug. 2-5, 2010 AIAA 2010-8373.

[12] W. Hu and D.J. Scheeres. Spacecraft motion about slowly rotating asteroids. Journal of Guidance, 
Control and Dynamics, 25(4), July-August 2002.

[13] W. Hu and D.J. Scheeres. Numerical determination of stability regions for orbital motion in uniformly rotating second degree and order gravity fields. Planetary and Space Science, Elsevier, 52:685-692, 2004.

[14] W. Hu and D.J. Scheeres. Periodic orbits in rotating second degree and order gravity fields. Chinese Journal of Astronomy and Astrophysics, 8(1):108-118, 2008.

[15] D.J. Scheeres, S.J. Ostro, R.S. Hudson, and R.A. Werner. Orbits close to asteroid 4769 Castalia. Icarus, 121:67-87, 1996.

[16] P. Geissler,J.M. Petit, D.D. Durda R. Greenberg, W. Bottke and M. Nolanz. Erosion and ejecta redistribution on 243 Ida and its moon. Icarus, 120:140-157, 1996.

[17] R.A. Werner and D.J. Scheeres. Exterior gravitation of a polyhedron derived and compared with harmonic and mascon gravitation representations of asteroid 4769 Castalia. Celestial Mechanics and Dynamical Astronomy, 65:31, 1996.

[18] G. Gómez, À. Jorba, J.J. Masdemont, and C. Simó. Dynamics and Mission Design Near Libration Point Orbits, volume 3 of Advanced Methods for Collinear Points. World Scientific, 2000.

[19] G. Gómez and J.M. Mondelo. The dynamics around the collinear equilibrium points of the rtbp. Physica D., (157):283-321, 2001.

[20] W.A. Heiskanen and H. Moritz. Physical Geodesy. W. H. Freeman and Company, San Francisco, USA, 1967.

[21] W.D. MacMillan. The Theory of Potential. Theoretical Mechanics. Dover Publications Inc., New York, USA, 1958.

[22] J.M.A. Danby. Fundamentals of Celestial Mechanics. The Macmillan Company, New York, USA, 1962.

[23] O.D. Kellog. Foundations of Potential Theory. Frederick Ungar Publishing Company, New York,USA, 1929.

[24] D.J. Scheeres. Stability of the planar full 2-body problem. Celestial Mechanics and Dynamical Astronomy, 104:103-128, 2009.

[25] A.J. Allen, P.L. Palmer, and J.Papaloizou. A conservative numerical technique for collisionless dynamical systems: comparison of the radial and circular orbit instabilities. Mon. Not. R. astr. Soc, 242:576-594, 1990.

[26] P.L. Palmer. Stability of collisionless stellar systems; mechanisms for the dynamical structure of galaxies, volume 185 of Astrophysics and Space Science Library. Kluwer Academic Publishers, Netherlands, 1994.

[27] D.J. Scheeres, S.J. Ostro, R.S. Hudson, E.M. DeJong, and S.Suzuki. Dynamics of orbits close to asteroid 
4179 Toutatis. Icarus, 132:53-79, 1998.

[28] D.J. Scheeres, B.G. Williams, and J.K. Miller. Evaluation of the dynamic environment of an asteroid: Application to 433 Eros. Journal of Guidance, Control and Dynamics, 23(3):466-475, May-June 2000.

[29] B.G Williams. Technical challenges and results for navigation of Near Shoemaker. John Hopkins APL Technical Digest, 23(1), 2002. 